\title{
CONTRACTIVE PROJECTIONS AND OPERATOR SPACES
}

\author{
MATTHEW NEAL AND BERNARD RUSSO
}

\begin{abstract}
Parallel to the study of finite dimensional Banach spaces, there is a growing interest in the corresponding local theory of operator spaces. We define a family of Hilbertian operator spaces $H_{n}^{k}, 1 \leq k \leq n$, generalizing the row and column Hilbert spaces $R_{n}, C_{n}$ and show that an atomic subspace $X \subset$ $B(H)$ which is the range of a contractive projection on $B(H)$ is isometrically completely contractive to an $\ell^{\infty}$-sum of the $H_{n}^{k}$ and Cartan factors of types 1 to 4 . In particular, for finite dimensional $X$, this answers a question posed by Oikhberg and Rosenthal. Explicit in the proof is a classification up to complete isometry of atomic $\mathrm{w}^{*}$-closed $J W^{*}$-triples without an infinite dimensional rank $1 \mathrm{w}^{*}$-closed ideal.
\end{abstract}

\section{Introduction}

It was shown by Choi-Effros that an injective operator system is isometric to a conditionally complete $C^{*}$-algebra [6, Theorem 3.1]. The fact that an injective operator system is the same as the image of a completely positive unital projection on $B(H)$ prompted a search for some algebraic structure in the range of a positive projection, or of a contractive projection. A special case of a result of Effros-Stormer showed that if a projection on a unital $C^{*}$-algebra is positive and unital, then the range is isometric to a Banach Jordan algebra [11, Theorem 1.4]. Arazy-Friedman (1) classified, up to Banach isometry, and in Banach space terms, the range of an arbitrary contractive projection on the $C^{*}$-algebra of all compact operators on a separable Hilbert space. A special case of a result of Friedman-Russo showed that if a projection on a $C^{*}$-algebra is contractive, then the range is isometric to a Banach Jordan triple system [13, Theorem 2]. Kaup [22] extended the Friedman-Russo result to contractive projections on $J B^{*}$-triples.

A consequence of these results is that, up to isometry, the ranges of the various projections can be classified modulo a classification theorem of the various algebraic structures involved. Recently, the operator space structure of the range of a completely contractive projection has been studied. For projections acting on $B(H)$, such spaces coincide with injectives in the category of operator spaces. Christensen and Sinclair [7, Theorem 1.1] prove that every injective von Neumann algebra with separable predual which is not finite type I of bounded degree is completely boundedly isomorphic to $B(H)$. Robertson and Wasserman [31, Corollary 7] prove that an infinite dimensional injective operator system on a separable Hilbert space is completely boundedly isomorphic to either $B(H)$ or $\ell^{\infty}$. Robertson and Youngson [32. Theorem 1] prove that every injective operator space is Banach isomorphic to one of $B(H), \ell^{\infty}, \ell^{2}$ or to a direct sum of these spaces. Robertson 30, Corollary

1991 Mathematics Subject Classification. Primary: 17C65. Secondary: 46L07.

Key words and phrases. Contractive projection, operator space, complete contraction, Cartan factor, injective, mixed-injective, $J C^{*}$-triple, $J W^{*}$-triple, ternary algebra.

This work was supported in part by NSF grant DMS-0101153. 
$3]$ proves that an injective operator space which is isometric to $\ell^{2}$ is completely isometric to $R$ or $C$ where $R$ and $C$ denote the row and column operator space versions of $\ell^{2}$. These results can be thought of as giving a partial classification of injectives up to various types of isomorphisms.

Note that the word injective in these examples is what we call 1-injective below. Also, the spaces appearing in the above results are all examples of atomic $J W^{*}$ triples, but only $\ell^{\infty}$ and $B(H)$ are $C^{*}$-algebras. Moreover, $B(H), R$ and $C$ are examples of Cartan factors, while $\ell^{\infty}$ is a direct sum of countably many copies of the trivial Cartan factor $\mathbb{C}$.

Operator spaces, that is, linear subspaces of $B(H)$, are the appropriate setting for these types of problems. They were first studied systematically in the thesis of Ruan [33] and have been developed extensively since then by Effros, Ruan, Blecher, Paulsen, Pisier, and others. Ruan [34, Theorem 4.5] showed that an operator space is injective if and only if it is completely isometric to $p A q$ for some injective $C^{*}$ algebra $A$ and projections $p, q \in A$. Youngson had shown earlier that the range of a completely contractive projection on a $C^{*}$-algebra is completely isometric to a ternary algebra, that is, a subspace of a $C^{*}$-algebra that is closed under the triple product $a b^{*} c[38$, Corollary 1].

Except for [1], there seem to be no results in the literature that classify the range of a contractive projection up to Banach isometry, or up to completely bounded isomorphism. In this paper, we remedy this by investigating the structure of operator spaces which are the range of a contractive projection on $B(H)$. These are known as 1-mixed injectives in operator space parlance. We provide in Theorem 2 a classification up to isometric complete contraction of 1-mixed injectives which are atomic. In Theorem 3, we classify up to complete isometry all atomic $\mathrm{w}^{*}$-closed $J W^{*}$-triples without an infinite dimensional rank $1 \mathrm{w}^{*}$-closed ideal. As a corollary, we show that an atomic (in particular, finite dimensional) contractively complemented subspace of a $C^{*}$-algebra is a 1-mixed injective, that is, the range of a contractive projection on some $B(H)$. Most of these results have been announced in [25].

\section{Preliminaries}

An operator space is a subspace $X$ of $B(H)$, the space of bounded linear operators on a complex Hilbert space. Its operator space structure is given by the sequence of norms on the set of matrices $M_{n}(X)$ with entries from $X$, determined by the identification $M_{n}(X) \subset M_{n}(B(H))=B(H \oplus H \oplus \cdots \oplus H)$. For the basic theory of operator spaces and completely bounded maps, we refer to [4], [9], [10], [28], and 29 and the references therein. Let us just recall that a linear mapping $\varphi: X \rightarrow Y$ between two operator spaces is completely bounded if the induced mappings $\varphi_{n}$ : $M_{n}(X) \rightarrow M_{n}(Y)$ defined by $\varphi_{n}\left(\left[x_{i j}\right]\right)=\left[\varphi\left(x_{i j}\right)\right]$ satisfy $\|\varphi\|_{\mathrm{cb}}:=\sup _{n}\left\|\varphi_{n}\right\|<\infty$. A completely bounded map is a completely bounded isomorphism if its inverse exists and is completely bounded. Two operator spaces are completely isometric if there is a linear isomorphism $T$ between them with $\|T\|_{\mathrm{cb}}=\left\|T^{-1}\right\|_{\mathrm{cb}}=1$. We call $T$ a complete isometry in this case.

In the matrix representation for $B\left(\ell^{2}\right)$ consider the column Hilbert space $C=$ $\overline{\mathrm{sp}}\left\{e_{i 1}: i \geq 1\right\}$ and the row Hilbert space $R=\overline{\mathrm{sp}}\left\{e_{1 j}: j \geq 1\right\}$ and their finite dimensional versions $C_{n}=\operatorname{sp}\left\{e_{i 1}: 1 \leq i \leq n\right\}$ and $R_{n}=\operatorname{sp}\left\{e_{1 j}: 1 \leq j \leq n\right\}$. 
Here of course $e_{i j}$ is the operator defined by the matrix with a 1 in the $(i, j)$ entry and zeros elsewhere. Although $R$ and $C$ are Banach isometric, they are not completely isomorphic; and $R_{n}$ and $C_{n}$, while completely isomorphic, are not completely isometric.

An operator space $Z$ is injective if for any operator space $Y$ and closed subspace $X \subset Y$, every completely bounded linear map $T: X \rightarrow Z$ has a completely bounded extension $\tilde{T}: Y \rightarrow Z$. In this case, there is a constant $\lambda \geq 1$ such that $\|\tilde{T}\|_{c b} \leq \lambda\|T\|_{c b}$ and $Z$ is said to be $\lambda$-injective. If $\lambda=1$, then $Z$ is also called isometrically injective. A fundamental theorem in operator space theory is that $B(H)$ is 1-injective. This is the celebrated Arveson-Wittstock Hahn-Banach Theorem, see [9, section 3]. It follows that an operator space $X \subset B(H)$ is $\lambda$ injective if and only if there is a completely bounded projection $P$ from $B(H)$ onto $X$ with $\|P\|_{c b} \leq \lambda$.

The literature on injective operator spaces cited in the introduction involves Cartan factors. Cartan factors appeared in the classification of Jordan triple systems and bounded symmetric domains. There are six types of Cartan factors of which four will be relevant to our work. A Cartan factor of type 1 is the space $B(H, K)$ of all bounded operators from one complex Hilbert space $H$ to another $K$. By fixing orthonormal bases for $H$ and $K$, we may think of $B(H, K)$ as all $\operatorname{dim} K$ by $\operatorname{dim} H$ matrices which define bounded operators. To define the Cartan factors of types 2 and 3 we need to fix a conjugation $J$ on a Hilbert space $H$, that is, a conjugatelinear isometry of order 2 . Then a Cartan factor of type 2 (respectively type 3 ) is $A(H, J)=\left\{x \in B(H): x^{t}=-x\right\}$ (respectively $S(H, J)=\left\{x \in B(H): x^{t}=x\right\}$ ), where $x^{t}=J x^{*} J$. Since conjugations are in one-to-one correspondence with orthonormal bases of $H$, we may think of these as anti-symmetric (resp. symmetric) $\operatorname{dim} H$ by $\operatorname{dim} H$ matrices which define bounded operators. A Cartan factor of type 4, or spin factor will be described in more detail in subsection 3.1.

The following concepts were introduced by Oikhberg and Rosenthal in 27, section 3] in their study of extension properties for the space of compact operators. The operator space $Z$ is a mixed injective if for every completely bounded linear map $T$ from an operator space $X$ into $Z$ and any operator space $Y$ containing $X$, $T$ has a bounded extension $\tilde{T}$ to $Y$. In this case, there is a constant $\lambda \geq 1$ such that $\|\tilde{T}\| \leq \lambda\|T\|_{c b}$, and $Z$ is said to be $\lambda$-mixed injective. A 1-mixed injective operator space is also said to be isometrically mixed injective and $X$ is $\lambda$-mixed injective if and only if there is a bounded projection $P$ from $B(H)$ onto $X$ with $\|P\| \leq \lambda$. An operator space $X$ is completely semi-isomorphic to an operator space $Y$ if there is a linear homeomorphism $T: X \rightarrow Y$ which is completely bounded. Such a $T$ is called a complete semi-isomorphism. If in addition $\|T\|_{c b}=\left\|T^{-1}\right\|=1$, then $X$ is completely semi-isometric to $Y$ and $T$ is a complete semi-isometry. It is shown in [27, Proposition 3.9] that mixed injectivity is preserved by complete semi-isomorphisms in the sense that if $Y$ is a mixed injective, then so is $X$.

The Cartan factors of types 1 to 4 are examples of 1 -mixed injectives. This is obvious for types 1,2,3 and for type 4 it is proved in [11. Lemma 2.3]. Cartan factors of types 5 and 6 will play no role in this paper since neither is even isometric to a 1 mixed injective operator space. For if it were, it would follow from 13, Theorem 2] that it would be isometric to a $J C^{*}$-triple (defined below). This is impossible since they are well-known to be "exceptional" ( i.e., not triple isomorphic to the Jordan triple structure induced by an associative *-algebra, see [15, 2.8.5] for the Jordan algebra version of this), and surjective isometries coincide with triple isomorphisms 
(the latter is proved for $J C^{*}$-triples in [16]; for the more general class of $J B^{*}$-triples see [21] or [2, Lemma 1]). The space of compact operators on a separable Hilbert space is not a 1-mixed injective, but it seems to be an interesting open question whether it has the mixed separable extension property [27], that is, in the definition of mixed injective, only separable operator spaces $X \subset Y$ are considered.

In view of the relaxed definition of 1-mixed injectives, one cannot immediately expect a classification of them up to complete isometry (however, see Theorem 3). It is more natural to ask for a classification of 1-mixed injectives up to complete semi-isometry. In order to formulate our results precisely we recall some basic facts about $J C^{*}$-triples.

A $J C^{*}$-triple is a norm closed complex linear subspace $M$ of a $C^{*}$-algebra $A$ which is closed under the operation $a \mapsto a a^{*} a$. $J C^{*}$-triples were defined and studied (using the name $J^{*}$-algebra) as a generalization of $C^{*}$-algebras by Harris $\mid 16$ in connection with function theory on infinite dimensional bounded symmetric domains. By a polarization identity, any $J C^{*}$-triple is closed under the triple product

$$
(a, b, c) \mapsto\{a b c\}:=\frac{1}{2}\left(a b^{*} c+c b^{*} a\right),
$$

under which it becomes a Jordan triple system. In this paper, the notation $\{a b c\}$ will always denote the triple product (11). A linear map which preserves the triple product (1) will be called a triple homomorphism. Cartan factors are examples of $J C^{*}$-triples, as are $C^{*}$-algebras, and Jordan $C^{*}$-algebras.

A $J W^{*}$-triple is defined to be a $J C^{*}$-triple which is a dual space. It follows from [2, Corollary 9] that a $J W^{*}$-triple is isometric to a $J C^{*}$-triple which is weak operator closed.

Note that some of the results about $J C^{*}$-triples that we are going to cite were proven for the more general class of $J B^{*}$-triples. For example, [3, Theorem 2.1] shows that all preduals of a $J W^{*}$-triple are isometric. $J B^{*}$-triples, in and of themselves, will play no role in this paper, but the interested reader can consult [35] for a comprehensive survey from an operator algebra point of view.

A special case of a $J C^{*}$-triple is a ternary algebra, that is, a subspace of $B(H, K)$ closed under the ternary product $(a, b, c) \mapsto a b^{*} c$. A ternary homomorphism is a linear map $\phi$ satisfying $\phi\left(a b^{*} c\right)=\phi(a) \phi(b)^{*} \phi(c)$. These spaces are also called, more appropriately, associative triple systems. They have been studied both concretely in 17 and abstractly in 39]. We shall use the term ternary algebra in this paper, but we shall not need any special results about them, other than the well-known and simple fact that a ternary isomorphism between two ternary algebras is a complete isometry. A key step in our proof of Theorem 2 will be to extend a Banach isometry between two $J W^{*}$-triples to a ternary isomorphism of their ternary envelopes.

If $v$ is a partial isometry in a $J C^{*}$-triple $M \subset B(H, K)$, then the projections $l=v v^{*} \in B(K)$ and $r=v^{*} v \in B(H)$ give rise to (Peirce) projections $P_{k}(v): M \rightarrow$ $M, k=2,1,0$ as follows; for $x \in M$,

$$
P_{2}(v) x=l x r \quad, \quad P_{1}(v) x=l x(1-r)+(1-l) x r \quad, \quad P_{0}(v) x=(1-l) x(1-r) .
$$

These projections $P_{k}(v)$ are easily seen to have the following properties. They are contractive projections and their ranges, denoted by $M_{k}(v)$ are $J C^{*}$-subtriples of $M$ satisfying $M=M_{2}(v) \oplus M_{1}(v) \oplus M_{0}(v)$. They obey Peirce calculus, by which is meant

$$
\left\{M_{2}(v) M_{0}(v) M\right\}=\left\{M_{0}(v) M_{2}(v) M\right\}=0 \quad, \quad\left\{M_{i}(v) M_{j}(v) M_{k}(v)\right\} \subset M_{i-j+k}(v)
$$


where it is understood that $M_{i-j+k}(v)=\{0\}$ if $i-j+k \notin\{0,1,2\}$.

The Peirce space $M_{2}(v)$ plays a special role. It has the structure of a unital Jordan ${ }^{*}$-algebra with unit $v$ under the product $(a, b) \mapsto a \circ b:=\{a v b\}$ and involution $a \mapsto a^{\sharp}:=\{v a v\}$. For example, the Jordan identity $(a \circ a) \circ(a \circ b)=a \circ((a \circ a) \circ b)$ amounts to $\{a v\{\{a v a\} v b\}\}=\{\{a v a\} v\{a v b\}\}$, which is trivial to verify for $J C^{*}$ triples. For more general Jordan triple systems see for example [24, 3.13], 23], or 37. 19.7], which are references for the general theory of (Banach) Jordan triple systems.

We shall write $M_{2}(v)^{(v)}$ to denote the space $M_{2}(v)$ with this structure. If $M=$ $M_{2}(v)$, then we refer to $M_{2}(v)^{(v)}$ as an isotope of $M$. If $M$ is a ternary algebra, then $M_{2}(v)^{(v)}$ is a unital $C^{*}$-algebra with product $a \cdot b=a v^{*} b$, involution $a^{\sharp}=v a^{*} v$, and unit $v$. In this case, the identity map from $M_{2}(v)$ to $M_{2}(v)^{(v)}$ is a ternary isomorphism, since $a b^{*} c=a v^{*} b^{\sharp} v^{*} c$, and hence also a complete isometry.

A partial isometry $v$ is said to be minimal in $M$ if $M_{2}(v)=\mathbb{C} v$. This is equivalent to $v$ not being the sum of two orthogonal non-zero partial isometries. Recall that two partial isometries $v$ and $w$ (or any two Hilbert space operators) are orthogonal if $v^{*} w=v w^{*}=0$. This is equivalent to $v \in M_{0}(w)$ and will be denoted by $v \perp w$. Each finite dimensional $J C^{*}$-triple is the linear span of its minimal paritial isometries. More generally, an atomic $J W^{*}$-triple is defined to be one which is the weak*-closure of the span of its minimal partial isometries. The rank of a $\mathrm{JC}^{*}$ triple is the maximum number of mutually orthogonal minimal partial isometries. For example, the rank of the Cartan factor of type $1 B(H, K)$ is the minimum of the dimensions of $H$ and $K$; and the rank of the Cartan factor of type 4 is 2. Other relations between two partial isometries that we shall need are defined in terms of the Peirce spaces as follows. Two partial isometries $v$ and $w$ are said to be colinear if $v \in M_{1}(w)$ and $w \in M_{1}(v)$, notation $v \top w$. A partial isometry $w$ is said to govern $v$ if $v \in M_{2}(w)$ and $w \in M_{1}(v)$. It is easy to check that $v \in M_{j}(w)$ if and only if $\{w w v\}=(j / 2) v$, for $j=0,1,2$.

$J C^{*}$-triples of arbitrary dimension occur naturally in functional analysis and in holomorphy. As noted in the introduction, a special case of a theorem of Friedman and Russo [13, Theorem 2] states that if $P$ is a contractive projection on a $C^{*}$-algebra $A$, then there is a linear isometry of the range $P(A)$ of $P$ onto a $J C^{*}$ subtriple of $A^{* *}$. A special case of a theorem of Kaup [21] gives a bijective correspondence between Cartan factors and irreducible bounded symmetric domains in complex Banach spaces.

There is a structure theorem for atomic $J W^{*}$-triples, for which we refer to 8 , p. 302], 18], 26] for the proofs. A $J W^{*}$-triple is irreducible if it is not the $\ell^{\infty}$-direct sum of 2 non-zero $w^{*}$-closed ideals. The version of the structure theorem that we shall use is the following.

Lemma 1.1. Each atomic $J W^{*}$-triple $X$ is the $\ell^{\infty}$-direct sum $X=\oplus_{\lambda}^{\ell^{\infty}} X_{\lambda}$ of weak-* closed irreducible ideals, and each summand $X_{\lambda}$ is the weak ${ }^{*}$-closure of the complex linear span of a grid of minimal partial isometries. Grids come in four types and each $X_{\lambda}$ is Banach isometric and hence triple isomorphic to a Cartan factor of one of the types 1-4.

We shall describe the grids for the Cartan factors of types 1-4 (the so-called rectangular grid, symplectic grid, hermitian grid, and spin grid) when they are needed later in this paper. As will be seen, grids only give information about the 
symmetrized triple product (11), whereas the operator space structure depends on the ternary product $(a, b, c) \mapsto a b^{*} c$.

It follows from Lemma 1.1] and [13, Theorem 2] that a finite dimensional 1-mixed injective operator space is Banach isometric to an $\ell^{\infty}$-direct sum of Cartan factors. Oikhberg and Rosenthal [27, Problem 3.3] ask whether every finite dimensional 1mixed injective operator space is in fact completely semi-isometric to an $\ell^{\infty}$-direct sum of Cartan factors of types 1-4. Corollary 2.1 of Theorem 2 below answers this question.

Theorem 2 below is formulated for atomic 1-mixed injective operator spaces. A Banach space $X$ with predual $X_{*}$ is said to be atomic if the closed unit ball $X_{*, 1}$ is the norm closed convex hull of its extreme points. In particular, reflexive Banach spaces are atomic as are the duals of unital $C^{*}$-algebras.

\section{Main Results and Reduction}

In this section we state Theorems 1,2, and 3, and give a reduction for the proof of Theorem 2 .

Theorem 1. There is a family of 1-mixed injective Hilbertian operator spaces $H_{n}^{k}$, $1 \leq k \leq n$, of finite dimension $n$, with the following properties:

(a): $H_{n}^{k}$ is a subtriple of the Cartan factor of type 1 consisting of all $\left(\begin{array}{l}n \\ k\end{array}\right)$ by $\left(\begin{array}{c}n \\ n-k+1\end{array}\right)$ complex matrices.

(b): Let $Y$ be a $J W^{*}$-triple of rank 1 (necessarily atomic ).

(i): If $Y$ is of finite dimension $n$ then it is isometrically completely contractive to some $H_{n}^{k}$.

(ii): If $Y$ is infinite dimensional then it is isometrically completely contractive to $B(H, \mathbb{C})$ or $B(\mathbb{C}, K)$.

(c): $H_{n}^{n}$ (resp. $\left.H_{n}^{1}\right)$ coincides with $R_{n}$ (resp. $\left.C_{n}\right)$.

(d): For $1<k<n, H_{n}^{k}$ is not completely semi-isometric to $R_{n}$ or $C_{n}$.

The spaces $H_{n}^{k}$ are explicitly constructed in section 6 . These spaces appeared in a slightly different form in [1], see Remark 7.6. The authors are grateful to N. Ozawa for showing us the proof of (d).

Theorem 2. Let $X$ be a 1-mixed injective operator space which is atomic. Then $X$ is completely semi-isometric to a direct sum of Cartan factors of types 1 to 4 and the spaces $H_{n}^{k}$.

The following Corollary to Theorem 2, together with (d) of Theorem 1, answers the question of Oikhberg and Rosenthal [27, Problem 3.3].

Corollary 2.1. A finite dimensional 1-mixed injective operator space is completely semi-isometric to a direct sum of Cartan factors of types 1 to 4 and the spaces $H_{n}^{k}$.

We now begin the proofs of Theorems 1 and 2.

Let $X \subset B(H)$ be a 1-mixed injective operator space. Then there is a contractive projection on $B(H)$ with range $X$. By [13, Theorem 2], there is thus a linear isometry $\mathcal{E}_{0}$ from $X$ onto a $J C^{*}$-triple $Y \subset A:=B(H)^{* *}$ of the form $\mathcal{E}_{0}(x)=p x q$ for suitable projections $p, q$ in the von Neumann algebra $A$. Since $\left(\mathcal{E}_{0}\right)_{n}: M_{n}(X) \rightarrow$ $M_{n}(Y)$ has the form $\left[x_{i j}\right] \mapsto \operatorname{diag}(p, p, \ldots, p)\left[x_{i j}\right] \operatorname{diag}(q, q, \ldots, q)$ we have the following lemma. 
Lemma 2.2. $\mathcal{E}_{0}$ is completely contractive and hence a complete semi-isometry of $X$ onto the $J C^{*}$-triple $Y$.

Lemma 2.3. Suppose $X$ is a Banach space with predual which is isometric to a $J C^{*}$-triple $Y$. Then $X$ is atomic as a Banach space if and only if $Y$ is an atomic $J W^{*}$-triple.

Proof. Since $X_{*}$ is a predual of $Y, Y$ is a $\mathrm{JW}^{*}$-triple. Assume $X$ is atomic as a Banach space. It is shown in [14, Prop 4c] that the minimal partial isometries $v$ in $Y$ are in 1-1 correspondence with extreme points $\phi$ of $Y_{*, 1}$ via the mapping $\phi \rightarrow v$ if $\phi(v)=1$. By [14, Theorem 2] $Y$ has an internal $\ell^{\infty}$ direct sum decomposition $\mathcal{A} \oplus \mathcal{N}$ into $\mathrm{w}^{*}$-closed subtriples where $\mathcal{A}$ is and atomic and $\mathcal{N}$ contains no minimal partial isometries. It follows that $\mathcal{N}=\{0\}$ and $Y$ is atomic.

Conversely, if $Y$ is an atomic $J W^{*}$-triple, then by [14. Theorem 1], $Y_{*}=A \oplus^{\ell^{1}} N$, where $N$ has no extreme points. It follows that $N=\{0\}$.

Lemma 2.4. It suffices to prove Theorem 园 in the case that $Y\left(=\mathcal{E}_{0}(X)\right)$ is triple isomorphic to a Cartan factor.

Proof. By Lemma 1.1 and Lemma 2.3, $Y=\oplus_{\alpha} Y_{\alpha}$ is the internal $\ell^{\infty}$ direct sum of a family of subtriples $Y_{\alpha}$, each of which is triple isomorphic to a Cartan factor of one of the types 1-4.

Suppose that $T_{\alpha}: Y_{\alpha} \rightarrow Z_{\alpha}$ is a complete semi-isometry. Then $\oplus T_{\alpha}: \oplus Y_{\alpha} \rightarrow$ $\oplus Z_{\alpha}$ is also a complete semi-isometry, by the following commutative diagram:

$$
\begin{array}{ccc}
M_{n}\left(\oplus Y_{\alpha}\right) & \stackrel{\text { isometry }}{\longrightarrow} & \oplus M_{n}\left(Y_{\alpha}\right) \\
\left(\oplus T_{\alpha}\right)_{n} \downarrow & \downarrow \oplus\left(T_{\alpha}\right)_{n} . \\
M_{n}\left(\oplus Z_{\alpha}\right) & \stackrel{\text { isometry }}{\longrightarrow} & \oplus M_{n}\left(Z_{\alpha}\right)
\end{array}
$$

To show the isometry part of the above diagram, one can use the idea of 114, Lemma 1.3]. For completeness, we include the argument. For $c \in M_{n}(X \oplus Y)$ with $c_{i j}=a_{i j} \oplus b_{i j}$, we have $c=\left[c_{i j}\right]=\left[a_{i j} \oplus 0\right]+\left[0 \oplus b_{i j}\right]=a+b$ with $a, b$ orthogonal operators, that is, $a b^{*}=a^{*} b=0$. Then, assuming $\|a\| \leq 1$ and $\|b\| \leq 1,\|c\|=\| a+$ $b\|=\|(a+b)(a+b)^{*}(a+b)\left\|^{1 / 3}=\right\| a^{3^{n}}\left\|^{3^{-n}}=\left(\left\|a^{3^{n}}+b^{3^{n}}\right\|^{3^{-n}}\right)^{3^{-n}} \leq\right\| a\left\|^{3^{n}}+\right\| b \|^{3^{n}} \leq$

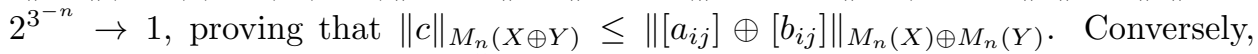
assume $\|a\|=1$. Then $1=\|a\|^{5}=\left\|a a^{*} a a^{*} a\right\|=\left\|a a^{*}(a+b) a^{*} a\right\| \leq\|a+b\|$, so that $\|a\| \leq\|c\|$ and it follows that $\left\|\left[a_{i j}\right] \oplus\left[b_{i j}\right]\right\|_{M_{n}(X) \oplus M_{n}(Y)} \leq\|c\|_{M_{n}(X \oplus Y)}$.

An $i d e a l$ of a $J C^{*}$-triple $Y$ is a subspace $I \subseteq Y$ such that $\left\{\begin{array}{lll}Y & I\end{array}\right\}+\left\{\begin{array}{lll}I & Y\end{array}\right\} \subseteq I$. By [12, Prop. 2.1], the second dual of a $J C^{*}$-triple is a $J W^{*}$-triple.

Lemma 2.5. Every $J W^{*}$-subtriple $Y$ of a $C^{*}$-algebra $A$ is completely semi-isometric to a $w^{*}$-closed $J W^{*}$-subtriple of $A^{* *}$.

Proof. By separate $\mathrm{w}^{*}$-continuity of multiplication, the annihilator $Y_{*}^{0}$ is a $\mathrm{w}^{*}$ closed ideal of $Y^{* *}$. By [18] or [26, Theorem 3.5], $Y^{* *}=Y_{*}^{0} \oplus^{\ell^{\infty}} J$, where $J$ is a $\mathrm{w}^{*}$-closed ideal orthogonal to $Y_{*}^{0}$. Let $P$ (resp. $Q$ ) be the projection of $Y^{* *}$ onto $Y_{*}^{0}\left(\right.$ resp. $J$ ). For each element $z \in Y^{* *}$, there is $y \in Y$ with $z-y \in Y_{*}^{0}$. It follows that $Q(Y)=Q\left(Y^{* *}\right)=J$, and it is easy to see by the orthogonality that $Q$ is a $\mathrm{w}^{*}$-continuous triple homomorphism from $Y^{* *}$ onto $J$. Since $P\left(Y^{* *}\right)=Y_{*}^{0}$, 
$Q$ is one-to-one on $Y$. As in the proof of Lemma 2.4, $Q=0 \oplus I d_{J}$ is a complete contraction.

Theorems 1(b) and 2 are immediate consequences of Lemmas 2.2, 2.4, 2.5 and the following proposition. Theorem $\mathbb{1}(\mathrm{a})$ is proved in section 6 (see Remark 6.2). Theorem $\mathbb{1}(\mathrm{c})$ is proved in Proposition 5.10 and Theorem $\mathbb{1}(\mathrm{d})$ is proved at the end of section 7 .

Proposition 2.6. Let $Y$ be a JW*-triple which is $w^{*}$-closed in a $W^{*}$-algebra. If $Y$ is either of rank at least 2, or of rank 1 and infinite dimensional, and is triple isomorphic to a Cartan factor of type 1,2,3, or 4, then it is in fact completely semiisometric to a Cartan factor of the same type. A finite dimensional JW*-triple which is triple isomorphic to a Cartan factor of rank 1, is completely semi-isometric to one of the spaces $H_{n}^{k}$.

As a by-product of the proof of Proposition 2.6, we shall obtain the following theorem, which by Lemma 1.1 gives a classification up to complete isometry of atomic $J W^{*}$-triples which are $\mathrm{w}^{*}$-closed and contain no infinite dimensional $\mathrm{w}^{*}$ closed ideals of rank 1.

We need the following definitions. If $B(H, K)$ is a Cartan factor of type 1 , then

$$
\operatorname{Diag}(B(H, K), B(K, H)):=\left\{\left(x, x^{t}\right): x \in B(H, K)\right\},
$$

where the transpose is respect to fixed orthonormal bases for $H$ and $K$. We give $\operatorname{Diag}(B(H, K), B(K, H))$ the operator space structure induced by its natural embedding in $B(H \oplus K \oplus H \oplus K)$ and note that $\operatorname{Diag}(B(H, K), B(K, H))$ is contractively complemented therein. Indeed, first project $B(H \oplus K \oplus H \oplus K)$ onto $B(H, K) \oplus B(K, H)$ and follow by $(x, y) \mapsto\left(\left(x+y^{t}\right) / 2,\left(x^{t}+y\right) / 2\right)$.

For a fixed dimension $n$ and each $j=1, \ldots, m$ let $H_{j}$ be a Hilbert space of dimension $n$ with a specified orthonormal basis $\mathcal{B}_{j}=\left\{e_{j, 1}, \ldots, e_{j, n}\right\}$. Then

$$
\operatorname{Diag}\left(\left\{H_{j}, \mathcal{B}_{j}\right\}\right):=\left\{\left(\sum_{k} \alpha_{k} e_{1 k}, \sum_{k} \alpha_{k} e_{2 k}, \ldots, \sum_{k} \alpha_{k} e_{m n}\right): \alpha_{k} \in \mathbb{C}, 1 \leq k \leq m\right\}
$$

The space Diag $\left(\left\{H_{j}, \mathcal{B}_{j}\right\}\right)$ is contractively complemented in $\oplus_{j=1}^{n} H_{j}$.

Theorem 3. Let $Y$ be an atomic $w^{*}$-closed $J W^{*}$-subtriple of a $W^{*}$-algebra.

(a): If $Y$ is irreducible and of rank at least 2, then it is completely isometric to a Cartan factor of type 1-4 or the space Diag $(B(H, K), B(K, H))$.

(b): If $Y$ is of finite dimension $n$ and of rank 1, then it is completely isometric to Diag $\left(H_{n}^{k_{1}}, \ldots, H_{n}^{k_{m}}\right)$, for appropriately chosen bases defined in section 7 , and where $k_{1}>k_{2}>\cdots>k_{m}$.

(c): $Y$ is completely semi-isometric to a direct sum of the spaces in (a) and (b). If $Y$ has no infinite dimensional rank 1 summand, then it is completely isometric to a direct sum of the spaces in (a) and (b).

Corollary 2.7. Every finite dimensional $J C^{*}$-triple is completely isometric to an $\ell^{\infty}$-direct sum of Cartan factors of types 1-4 and the spaces Diag $(B(H, K), B(K, H))$ and $\operatorname{Diag}\left(H_{n}^{k_{1}}, \ldots, H_{n}^{k_{m}}\right)$.

Corollary 2.8. Every atomic contractively complemented subspace $X$ of a $C^{*}$ algebra $A$ is 1-mixed injective. 
Proof. By [13, Theorem 2] and Lemma 2.3, the map $\mathcal{E}_{0}$ mentioned earlier is a complete semi-isometry of $X$ onto an atomic $J W^{*}$-subtriple of $A^{* *}$. By Theorem 3 and Lemma 2.5, $X$ is completely semi-isometric to a direct sum of the spaces listed above, which as noted are 1-mixed injectives. Then by [27, (3.9)], $X$ is 1-mixed injective.

Corollary 2.9. Every atomic $J W^{*}$-triple is a 1-mixed injective.

We shall prove Proposition 2.6 and Theorem 3 case by case in the following sections. Cartan factors of Types 3 and 4 are handled in section 3, type 2 in section 4 and type 1 in sections 5,6 and 7. Section 6 also introduces the spaces $H_{n}^{k}$ and section 7 also gives some examples and states some open problems.

\section{CARTAN Factors of types 3 AND 4}

The Cartan factors of types 3 and 4 have a unital Jordan *-algebra structure in which we frame the proofs of Proposition 2.6 and Theorem 3.

3.1. Cartan factors of type 4. We first prove Proposition 2.6 and Theorem 3 in the case that $Y$ is triple isomorphic to a Cartan factor of type 4. Let us first describe the concrete model which we use for such a Cartan factor, from [15, Theorem 6.2.2] and [16].

A spin system is a subset $\mathcal{S}=\left\{1, s_{1}, \ldots, s_{k}\right\}$ of self-adjoint elements of $B(H)$ containing the unit and satisfying $s_{i} s_{j}+s_{j} s_{i}=\delta_{i j} 2$. It follows that $\operatorname{sp}_{\mathbb{C}} \mathcal{S}$ is a $(k+1)$-dimensional Jordan $C^{*}$-subalgebra of $B(H)$. A spin factor is a subspace $X$ of $B(H)$ of dimension at least 2 which is the closed linear span of a spin system of arbitrary cardinality.

We now recall the standard matrix representation of the spin factor $\operatorname{Sp}(n), 3 \leq$ $n \leq \infty$, for the separable case (c.f. [15, 6.2.1]), which is the Cartan factor of type 4 . Let

$$
\sigma_{1}=\left[\begin{array}{rr}
1 & 0 \\
0 & -1
\end{array}\right] \quad, \quad \sigma_{2}=\left[\begin{array}{ll}
0 & 1 \\
1 & 0
\end{array}\right] \quad, \quad \sigma_{3}=\left[\begin{array}{rr}
0 & i \\
-i & 0
\end{array}\right]
$$

be the Pauli spin matrices. Denote by $\sigma_{3}^{n}$ the $n$-fold tensor product $\sigma_{3} \otimes \cdots \otimes \sigma_{3}$ of $\sigma_{3}$ with itself $n$ times in $M_{2^{n}}(\mathbb{C})$. Define

$$
s_{1}=\sigma_{1}, s_{2}=\sigma_{2}, s_{3}=\sigma_{3} \otimes \sigma_{1}, s_{4}=\sigma_{3} \otimes \sigma_{2} \ldots
$$

and in general $s_{2 n+1}=\sigma_{3}^{n} \otimes \sigma_{1}$ and $s_{2 n+2}=\sigma_{3}^{n} \otimes \sigma_{2}$.

With the imbeddings $M_{2^{n}}(\mathbb{C}) \subset M_{2^{n+1}}(\mathbb{C})$ given by

$$
a \mapsto a \otimes 1=\left[\begin{array}{ll}
a & 0 \\
0 & a
\end{array}\right]
$$

we have $s_{k} \in M_{2^{n}}(\mathbb{C})$ if $k \leq 2 n$ and $\left\{1, s_{1}, \ldots, s_{k}\right\}$ is a spin system for each $k \geq 2$. The linear span $\mathrm{Sp}(k+1)$ of $\left\{1, s_{1}, \ldots, s_{k}\right\}$ is a $(k+1)$-dimensional spin factor contained in $M_{2^{n}}(\mathbb{C})$ if $2 \leq k \leq 2 n$. For more details and the case $k=\aleph_{0}$, see [15. Theorem 6.2.2].

As an operator space, a spin factor $X$ is determined up to complete isometry by the cardinality of the spin system. Indeed, it is easy to see that two finite spin systems with the same number of elements generate $C^{*}$-algebras which are ${ }^{*}$ isomorphic with basis consisting of all finite products of elements in the spin system and the unit. If a spin system $\left\{s_{\lambda}\right\}_{\lambda \in \Lambda}$ has arbitrary cardinality, the inductive limit $\overline{\cup_{F} A(F)}$ (norm closure) of the collection of $C^{*}$-algebras $A(F)$ generated by finite 
subsets $F$ of $\left\{s_{\lambda}\right\}_{\lambda \in \Lambda}$ is exactly the $C^{*}$-algebra generated by the spin system. Thus, any two spin systems with the same cardinality generate ${ }^{*}$-isomorphic $C^{*}$-algebras (cf. [36, 1.23] or [5, Theorem 5.2.5]).

Suppose now that the $Y$ in the statement of Proposition 2.6 is triple isomorphic to a Cartan factor of type 4 . Let $A$ denote any von Neumann algebra containing $Y$. The $J W^{*}$-triple $Y$ contains a spin grid $\left\{u_{j}, \tilde{u}_{j}: j \in J\right\}$, or $\left\{u_{j}, \tilde{u}_{j}: j \in J\right\} \cup\left\{u_{0}\right\}$ in the case that $Y$ is of finite odd dimension.

Let us recall the properties of a spin grid from [8, p. 313]. The elements $u_{j}$ and $\tilde{u}_{j}$ (but not $u_{0}$ ) are minimal non-zero partial isometries; for $i \neq j, u_{i}$ is colinear with $u_{j}$ and with $\tilde{u}_{j}$, and $\tilde{u}_{j}$ is colinear with $\tilde{u}_{i}$; and for $i \neq j$,

$$
\left\{u_{i} u_{j} \tilde{u}_{i}\right\}=-\frac{1}{2} \tilde{u}_{j},\left\{u_{j} \tilde{u}_{i} \tilde{u}_{j}\right\}=-\frac{1}{2} \tilde{u}_{i}
$$

In case $u_{0}$ is present, for each $i \neq 0, u_{0}$ governs $u_{i}$ and $\tilde{u}_{i}$, and

$$
\left\{u_{0} u_{i} u_{0}\right\}=-\tilde{u}_{i},\left\{u_{0} \tilde{u}_{i} u_{0}\right\}=-u_{i}
$$

All other triple products from the spin grid are 0 , and in particular, $u_{i}$ is orthogonal to $\tilde{u}_{i}$.

It is not hard to see (c.f. \$ 8 ) that the complex span $Y$ of a spin grid has an equivalent Hilbertian norm and is hence reflexive. It is also clear from the grid properties that all such $Y$ are rank 2 .

Let $v=i\left(u_{1}+\tilde{u}_{1}\right)$, where 1 is an arbitrary element of the index set $J$. It is easy to see that that $Y=Y_{2}(v)$. As noted in the preliminaries, $A_{2}(v)$ and $A_{2}(v)^{(v)}$ are ternary isomorphic and thus completely isometric. Thus, the identity map $Y \rightarrow Y_{2}(v)^{(v)}$ is a complete isometry.

The following lemma is easily verified by using (2), (3) and Peirce calculus. For the convenience of the reader, we include some of the details.

Lemma 3.1. $Y_{2}(v)^{(v)}$ is a Cartan factor of type 4. More precisely, let $s_{j}=u_{j}+$ $\tilde{u}_{j}, j \in J-\{1\} ; t_{j}=i\left(u_{j}-\tilde{u}_{j}\right), j \in J$. Then a spin system in the $C^{*}$-algebra $A_{2}(v)^{(v)}$ which linearly spans $Y_{2}(v)^{(v)}$ is given by

$$
\left\{s_{j}, t_{k}, v: j \in J-\{1\}, k \in J\right\}
$$

or, if the spin factor is of odd finite dimension,

$$
\left\{s_{j}, t_{k}, v, u_{0}: j \in J-\{1\}, k \in J\right\}
$$

Proof. If $j \neq 1, k \neq 1$,

$$
\begin{aligned}
s_{j} \cdot s_{k}+s_{k} \cdot s_{j} & =s_{j} v^{*} s_{k}+s_{k} v^{*} s_{j}=2\left\{s_{j} v s_{k}\right\} \\
& =-2 i\left\{u_{j}+\tilde{u}_{j}, u_{1}+\tilde{u}_{1}, u_{k}+\tilde{u}_{k}\right\}
\end{aligned}
$$

If $j \neq k$ then all 8 terms in the expansion of this triple product are zero since the triple product of three mutually colinear partial isometries is zero. On the other hand,

$$
\begin{aligned}
2 s_{j} \cdot s_{j} & =-2 i\left\{u_{j}+\tilde{u}_{j}, u_{1}+\tilde{u}_{1}, u_{j}+\tilde{u}_{j}\right\} \\
& =-2 i\left[\left\{u_{j} u_{1} u_{j}\right\}+\left\{u_{j} u_{1} \tilde{u}_{j}\right\}+\left\{u_{j} \tilde{u}_{1} u_{j}\right\}+\left\{u_{j} \tilde{u}_{1} \tilde{u}_{j}\right\}\right] \\
& +-2 i\left[\left\{\tilde{u}_{j} u_{1} u_{j}\right\}+\left\{\tilde{u}_{j} u_{1} \tilde{u}_{j}\right\}+\left\{\tilde{u}_{j} \tilde{u}_{1} u_{j}\right\}+\left\{\tilde{u}_{j} \tilde{u}_{1} \tilde{u}_{j}\right\}\right] \\
& \left.=-2 i\left[0-\tilde{u}_{1} / 2+0-u_{1} / 2-\tilde{u}_{1} / 2+0-u_{1} / 2+0\right] \text { (by (2) }\right) \\
& =2 v
\end{aligned}
$$


Similarly, $t_{j} \cdot t_{k}+t_{k} \cdot t_{j}=2 \delta_{j k} v$ for all $j, k \in J$; and $s_{j} \cdot t_{k}+t_{k} \cdot s_{j}=0$ for all $j \in J-\{1\}, k \in J$.

Next we consider the case that $u_{0}$ is present. If $j \neq 1$,

$$
\begin{aligned}
s_{j} \cdot u_{0}+u_{0} \cdot s_{j} & =s_{j} v^{*} u_{0}+u_{0} v^{*} s_{j}=2\left\{s_{j} v u_{0}\right\} \\
& =-2 i\left\{u_{j}+\tilde{u}_{j}, u_{1}+\tilde{u}_{1}, u_{0}\right\} \\
& =-2 i\left[\left\{u_{j} u_{1} u_{0}\right\}+\left\{u_{j} \tilde{u}_{1} u_{0}\right\}+\left\{\tilde{u}_{j} u_{1} u_{0}\right\}+\left\{\tilde{u}_{j} \tilde{u}_{1} u_{0}\right\}\right] .
\end{aligned}
$$

By Peirce calculus $\left\{u_{j} u_{1} u_{0}\right\}$ is orthogonal to $u_{1}$ and is a multiple of $u_{j}$, hence it is zero. Similarly, each of the other three terms is zero, and similarly $t_{j} \cdot u_{0}+u_{0} \cdot t_{j}=0$ for all $j \in J$. Finally $u_{0} \cdot u_{0}=-i\left\{u_{0}, u_{1}+\tilde{u}_{1}, u_{0}\right\}=v$ by (3).

As $Y$ is completely isometric to $Y_{2}(v)^{(v)}$, this completes the proof of Proposition 2.6 and Theorem 3 in the case that $Y$ is triple isomorphic to a Cartan factor of type 4 .

3.2. Cartan factors of type 3. We next prove Proposition 2.6 and Theorem 3 in the case that $Y$ is triple isomorphic to the Cartan factor $S(H, J)$, of type 3. Again, we let $A$ denote any von Neumann algebra containing $Y$.

Let us recall that an hermitian grid (cf. [8, p. 308]) is a family $\left\{u_{i j}: i, j \in I\right\}$ of partial isometries satisfying $u_{i j}=u_{j i} ; u_{i j} \perp u_{k l}$ if $\{i, j\} \cap\{k, l\}=\emptyset ; u_{i j} \vdash u_{i i}$ if $i \neq j ; u_{i j} \top u_{j k}$ if $i, j, k$ distinct; $\left\{u_{i j} u_{j k} u_{k l}\right\}=u_{i l} / 2$ and $\left\{u_{i j} u_{j k} u_{k i}\right\}=u_{i i}$ if $i \neq l$ and at least two of these elements are distinct; and all other triple products are 0 .

Let $\left\{u_{i j}: i, j \in \Lambda\right\}$ be an hermitian grid which is $\mathrm{w}^{*}$-total in $Y$ and let $v$ denote the partial isometry $\sum_{i} u_{i i}$ (the sum is $\mathrm{w}^{*}$-convergent since $u_{i i} \perp u_{j j}$ ) and note that it has the property that $Y=Y_{2}(v)$. Let $\psi: Y \rightarrow S(H, J)$ be the triple isomorphism determined by $\psi\left(u_{i j}\right)=U_{i j}$, where $\left\{U_{i j}\right\}$ denotes the canonical hermitian grid for $S(H, J)$, that is, $U_{i j}=\phi_{j} \otimes \phi_{i}+\phi_{i} \otimes \phi_{j}$ for $i \neq j$ and $U_{i i}=\phi_{i} \otimes \phi_{i}$ for an orthonormal basis $\left\{\phi_{\lambda}\right\}$ of $H$.

Note that isomorphisms of $J W^{*}$-triples (being isometries on spaces with unique preduals) are automatically $\mathrm{w}^{*}$-continuous. Hence $\psi(v)=I d_{H}$ and $\psi\left(u_{i j}^{\sharp}\right)=$ $\left\{\psi(v), \psi\left(u_{i j}\right), \psi(v)\right\}=\psi\left(u_{i j}\right)^{*}=U_{i j}^{*}=U_{i j}=\psi\left(u_{i j}\right)$, so that $u_{i j}$ is self-adjoint in $A_{2}(v)$. Here, $a^{\sharp}=v a^{*} v$ denotes the involution in $A_{2}(v)^{(v)}$. Also, recall that the ternary product is the same whether it is computed in $A$ or in $A_{2}(v)^{(v)}$, that is, $x y^{*} z=x v^{*}\left(v y^{*} v\right) v^{*} z$.

Now define $e_{i j}=u_{i i} \cdot u_{i j}$, where we use $a \cdot b$ to denote the associative product in $A_{2}(v)^{(v)}$, that is, $a \cdot b=a v^{*} b$.

Lemma 3.2. The collection $\left\{e_{i j}\right\}$ forms a system of matrix units in $A_{2}(v)^{(v)}$, that is,

(a): $e_{i j}^{\sharp}=e_{j i}, e_{i j} \cdot e_{k l}=\delta_{j k} e_{i l}, v=\sum e_{i i}$.

Moreover,

(b): $u_{i i} \cdot u_{i j}=u_{i j} \cdot u_{j j}$ and $u_{i j}=e_{i j}+e_{j i}$.

(c): $\psi$ extends to $a{ }^{*}$-isomorphism $\tilde{\psi}: s p_{\mathbb{C}}\left\{e_{i j}\right\} \rightarrow s p_{\mathbb{C}}\left\{E_{i j}\right\}$ satisfying $\tilde{\psi}\left(e_{i j}\right)=$ $E_{i j}$, where $E_{i j}=\phi_{j} \otimes \phi_{i}$.

Proof. We first show these three identities:

$$
\begin{gathered}
\left(u_{i i} \cdot u_{i j}-u_{i j} \cdot u_{j j}\right)^{\sharp}\left(u_{i i} \cdot u_{i j}-u_{i j} \cdot u_{j j}\right)=0, \\
\left(e_{i j} \cdot e_{k l}\right) \cdot\left(e_{i j} \cdot e_{k l}\right)^{\sharp}=0 \text { for } j \neq k,
\end{gathered}
$$




$$
\left(e_{i j} \cdot e_{j l}-e_{i l}\right) \cdot\left(e_{i j} \cdot e_{j l}-e_{i l}\right)^{\sharp}=0 .
$$

Note that $u_{i i} \cdot u_{i i}=u_{i i} v^{*} u_{i i}=u_{i i}\left(\sum_{j} u_{j j}^{*}\right) u_{i i}=u_{i i}$, and that similarly, for $i \neq j$, $u_{i j} \cdot u_{i j}=u_{i i}+u_{j j}, u_{i i} \cdot u_{j j}=0$, and $u_{i j} \cdot u_{i i} \cdot u_{i j}=u_{j j}$. Therefore, for $i \neq j$,

$$
\begin{aligned}
& \left(u_{i i} \cdot u_{i j}-u_{i j} \cdot u_{j j}\right)^{\#} \cdot\left(u_{i i} \cdot u_{i j}-u_{i j} \cdot u_{j j}\right)= \\
& =\left(u_{i j} \cdot u_{i i}-u_{j j} \cdot u_{i j}\right) \cdot\left(u_{i i} \cdot u_{i j}-u_{i j} \cdot u_{j j}\right) \\
& =u_{i j} \cdot\left(u_{i i} \cdot u_{i i}\right) \cdot u_{i j}-u_{j j} \cdot\left(u_{i j} \cdot u_{i i} \cdot u_{i j}\right) \\
& \text { - }\left(u_{i j} \cdot u_{i i} \cdot u_{i j}\right) \cdot u_{j j}+u_{j j} \cdot\left(u_{i j} \cdot u_{i j}\right) \cdot u_{j j} \\
& =u_{i j} \cdot u_{i i} \cdot u_{i j}-u_{j j} \cdot u_{j j}-u_{j j} \cdot u_{j j}+u_{j j} \cdot\left(u_{i i}+u_{j j}\right) \cdot u_{j j} \\
& =u_{j j}-u_{j j}-u_{j j}+u_{j j}=0 \text {, proving (4) and the first statement in (b). }
\end{aligned}
$$

Next, if $j \neq k$, then

$$
\begin{aligned}
\left(e_{i j} \cdot e_{k l}\right) \cdot\left(e_{i j} \cdot e_{k l}\right)^{\#} & =e_{i j} \cdot e_{k l} \cdot e_{l k} \cdot e_{j i} \\
& =u_{i j} \cdot u_{j j} \cdot\left(u_{k l} \cdot u_{l l} \cdot u_{l k}\right) \cdot u_{k k} \cdot u_{j i} \cdot u_{i i}(\mathrm{by}(\mathrm{b})) \\
& =u_{i j} \cdot u_{j j} \cdot\left(u_{k k} \cdot u_{k k}\right) u_{i j} \cdot u_{i i} \\
& =u_{i j} \cdot u_{j j} \cdot u_{k k} \cdot u_{i j} \cdot u_{i i}=0, \text { proving (5). }
\end{aligned}
$$

Next,

$$
\begin{aligned}
& \left(e_{i j} \cdot e_{j l}-e_{i l}\right) \cdot\left(e_{i j} \cdot e_{j l}-e_{i l}\right)^{\sharp}= \\
& \quad=\left(e_{i j} \cdot e_{j l}-e_{i l}\right) \cdot\left(e_{l j} \cdot e_{j i}-e_{l i}\right) \\
& =\left(u_{i j} \cdot u_{j j} \cdot u_{j l} \cdot u_{l l}-u_{i l} \cdot u_{l l}\right) \cdot\left(u_{l l} \cdot u_{j l} \cdot u_{j j} \cdot u_{i j}-u_{l l} \cdot u_{l i}\right) \\
& =-u_{i j} \cdot u_{j j} \cdot u_{j l} \cdot u_{l l} \cdot u_{l i}+u_{i l} \cdot u_{l l} \cdot u_{l i} \\
& \quad+u_{i j} \cdot u_{j j} \cdot\left(u_{j l} \cdot u_{l l} \cdot u_{j l}\right) \cdot u_{j j} \cdot u_{i j}-u_{i l} \cdot u_{l l} \cdot u_{j l} \cdot u_{j j} \cdot u_{i j} \\
& =-A+u_{i i}+u_{i j} \cdot u_{j j} \cdot u_{j j} \cdot u_{j j} \cdot u_{i j}-B \\
& =-A+u_{i i}+u_{i i}-B
\end{aligned}
$$

where $A=u_{i j} \cdot u_{j j} \cdot u_{j l} \cdot u_{l l} \cdot u_{l i}$ and $B=u_{i l} \cdot u_{l l} \cdot u_{j l} \cdot u_{j j} \cdot u_{i j}$.

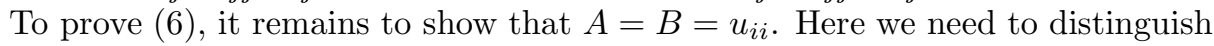
cases. Suppose first that $i, j$ and $l$ are distinct. Then $\left\{u_{j l} u_{l l} u_{i l}\right\}=u_{i j} / 2$ so that

$$
\begin{aligned}
A & =u_{i j} \cdot u_{j j} \cdot\left(2\left\{u_{j l} u_{l l} u_{i l}\right\}-u_{i l} \cdot u_{l l} \cdot u_{j l}\right) \\
& =u_{i j} \cdot u_{j j} \cdot u_{i j}-u_{i j} \cdot u_{j j} \cdot\left(u_{i l} \cdot u_{l l}\right) \cdot u_{j l} \\
& =u_{i i}-u_{i j} \cdot u_{j j} \cdot\left(u_{i i} \cdot u_{i l}\right) \cdot u_{j l} \text { (by the first statement in (b)) } \\
& =u_{i i} \text { as required. }
\end{aligned}
$$

Also $B=u_{i l} \cdot u_{l l} \cdot\left(2\left\{u_{j l} u_{j j} u_{i j}\right\}-u_{i j} \cdot u_{j j} \cdot u_{j l}\right)=u_{i l} \cdot u_{l l} \cdot u_{l i}-u_{i l} \cdot u_{l l} \cdot u_{i j} \cdot u_{j j} \cdot u_{j l}=u_{i i}$. Now if $i=j$, then $A=u_{i i} \cdot u_{i i} \cdot u_{i l} \cdot u_{l l} \cdot u_{i l}=u_{i i}^{3}=u_{i i}$ and $B=u_{i l} \cdot u_{l l} \cdot u_{i l} \cdot u_{i i} \cdot u_{i i}=$ $u_{i i}^{3}=u_{i i}$. Similarly if $l=j$ or $i=l$, proving (6).

Finally, since $u_{i j} u_{k k}=0$ if $k \notin\{i, j\}, e_{i j}+e_{j i}=u_{i j} \cdot u_{j j}+u_{i j} \cdot u_{i i}=u_{i j} \cdot\left(\sum u_{k k}\right)=$ $u_{i j}$. This completes the proof of (a) and (b).

By the first statement in (b), we have

$$
e_{i j}^{\sharp}=\left(u_{i i} \cdot u_{i j}\right)^{\sharp}=u_{i j} \cdot u_{i i}=u_{j j} u_{j i}=e_{j i} .
$$

Since the system of matrix units $\left\{e_{i j}\right\}$ are linearly independent, $\tilde{\psi}$ defines a linear isomorphism of $\operatorname{sp}_{\mathbb{C}}\left\{e_{i j}\right\}$ onto $\operatorname{sp} \mathbb{C}\left\{E_{i j}\right\}$ which is by construction a ${ }^{*}$-isomorphism, proving (c). 
Clearly $\tilde{\psi}$ extends to a ${ }^{*}$-isomorphism from the $C^{*}$-subalgebra $\overline{\operatorname{sp} \mathbb{C}\left\{e_{i j}\right\}}{ }^{\|\cdot\|}$ of $A_{2}(v)^{(v)}$ onto the $C^{*}$-algebra of compact operators $K(H)$. By [8, Lemma 1.14], $\tilde{\psi}$ extends to a $\mathrm{w}^{*}$-continuous isometry and, hence, ${ }^{*}$-isomorphism from $\overline{\operatorname{sp}_{\mathbb{C}}\left\{e_{i j}\right\}} \mathrm{w}^{*}$ onto $B(H)$. Since a ${ }^{*}$-isomorphism is completely isometric, the proof of Proposition 2.6 and Theorem 3 is completed in the case that $Y$ is triple isomorphic to a Cartan factor of type 3 .

\section{Cartan factors of type 2}

In this section, we prove Proposition 2.6 and Theorem 3 in the case that $Y$ is triple isomorphic to the Cartan factor $A(H, J)$ of type 2. Again, $A$ denotes any von Neumann algebra containing $Y$. Since $A\left(\mathbb{C}^{3}, J\right)$ is triple isomorphic to $B\left(\mathbb{C}, \mathbb{C}^{3}\right)$, which is covered in section 7 , and $A\left(\mathbb{C}^{4}, J\right)$ is triple isomorphic to $\mathrm{Sp}(6)$, which was covered in section 3 , we may and shall assume that $\operatorname{dim} H>4$.

Let us recall $\left(\mathbb{8}\right.$, p. 317] that a symplectic grid is a family $\left\{u_{i j}: i, j \in I, i \neq j\right\}$ of minimal partial isometries satisfying $u_{i j}=-u_{j i} ; u_{i j} \top u_{k l}$ if $\{i, j\} \cap\{k, l\} \neq \emptyset$; $u_{i j} \perp u_{k l}$ if $\{i, j\} \cap\{k, l\}=\emptyset ; 2\left\{u_{i j} u_{i l} u_{k l}\right\}=u_{k j}$ for distinct $i, j, k, l$; and all other triple products vanish. The fact that each $u_{i j}$ is minimal can be expressed by

$$
u_{i j} u_{k l}^{*} u_{i j}=\delta_{(i, j),(k, l)} u_{i j} \text {. }
$$

Let $\left\{u_{i j}\right\}$ be a symplectic grid which is w $^{*}$-total in $Y$. Let $\psi: Y \rightarrow A(H, J)$ be the triple isomorphism determined by $\psi\left(u_{i j}\right)=U_{i j}$, where $\left\{U_{i j}\right\}=\phi_{j} \otimes \phi_{i}-\phi i \otimes \phi_{j}$ for an orthonormal basis $\left\{\phi_{\lambda}\right\}$ of $H$.

Lemma 4.1. For any indices $i, j, k, l, m$,

$$
u_{i k} u_{k l}^{*} u_{i l}=u_{i j} u_{j m}^{*} u_{i m},
$$

and for $1 \leq i \leq n$, the elements $e_{i i}$ unambiguously defined by $e_{i i}=u_{i j} u_{j m}^{*} u_{i m}$ are non-zero orthogonal partial isometries in A.

Proof. We shall use repeatedly the fact that $u_{i j}=-u_{j i}$.

Suppose that $i, j, k, l$ are distinct. Then $u_{i j} u_{k l}^{*}=0$ and therefore

$$
\begin{aligned}
u_{i k} u_{k l}^{*} u_{i l} & =2\left\{u_{i j} u_{i j} u_{i k}\right\} u_{k l}^{*} u_{i l} \\
& =\left(u_{i j} u_{i j}^{*} u_{i k}+u_{i k} u_{i j}^{*} u_{i j}\right) u_{k l}^{*} u_{i l} \\
& =u_{i j} u_{i j}^{*} u_{i k} u_{k l}^{*} u_{i l}+0 \\
& =u_{i j}\left(u_{k l} u_{i k}^{*} u_{i j}+u_{i j} u_{i k}^{*} u_{k l}\right)^{*} u_{i l} \\
& =2 u_{i j}\left\{u_{i j} u_{i k} u_{k l}\right\}^{*} u_{i l} \\
& =u_{i j}\left(-u_{l j}\right)^{*} u_{i l}=u_{i j} u_{j l}^{*} u_{i l} .
\end{aligned}
$$

Similarly, if $m, l, i, k$ are distinct, by replacing $u_{i l}$ by $2\left\{u_{i m} u_{i m} u_{i l}\right\}$, we obtain $u_{i k} u_{k l}^{*} u_{i l}=u_{i k} u_{k m}^{*} u_{i m}$. Indeed,

$$
\begin{aligned}
u_{i k} u_{k l}^{*} u_{i l} & =u_{i k} u_{k l}^{*} 2\left\{u_{i m} u_{i m} u_{i l}\right\} \\
& =u_{i k} u_{k l}^{*}\left(u_{i m} u_{i m}^{*} u_{i l}+u_{i l} u_{i m}^{*} u_{i m}\right) \\
& =u_{i k} u_{k l}^{*} u_{i l} u_{i m}^{*} u_{i m} \\
& =2 u_{i k}\left\{u_{k l} u_{i l} u_{i m}\right\}^{*} u_{i m} \\
& =u_{i k} u_{k m}^{*} u_{i m} .
\end{aligned}
$$

Together, (9) and (10) show (8) and thus $e_{i i}$ is well defined. 
We next show that $e_{i i} \neq 0$. Suppose instead that $e_{i i}=0$ for some $i$. For $i, k, l$ distinct, $u_{i k} \top u_{k l}$ and $u_{k l} \top u_{i l}$, so

$$
\begin{aligned}
u_{k l} & =u_{i k} u_{i k}^{*} u_{k l}+u_{k l} u_{i k}^{*} u_{i k} \\
& =u_{i k} u_{i k}^{*}\left(u_{i l} u_{i l}^{*} u_{k l}+u_{k l} u_{i l}^{*} u_{i l}\right)+\left(u_{i l} u_{i l}^{*} u_{k l}+u_{k l} u_{i l}^{*} u_{i l}\right) u_{i k}^{*} u_{i k} \\
& \left.=\left(u_{i k} u_{i k}^{*} u_{i l} u_{i l}^{*} u_{k l}+u_{i k} e_{i i}^{*} u_{i l}\right)+\left(u_{i l} e_{i i}^{*} u_{i k}+u_{k l} u_{i l}^{*} u_{i l}\right) u_{i k}^{*} u_{i k}\right) \\
& \left.=\left(u_{i k} u_{i k}^{*} u_{i l} u_{i l}^{*} u_{k l}+0\right)+\left(0+u_{k l} u_{i l}^{*} u_{i l}\right) u_{i k}^{*} u_{i k}\right) \\
& =L_{i k} L_{i l} u_{k l}+u_{k l} R_{i l} R_{i k},
\end{aligned}
$$

where $L_{i k}=u_{i k} u_{i k}^{*}$ and $R_{i k}=u_{i k}^{*} u_{i k}$ denote the left and right support projections of $u_{i k}$.

By the definition of symplectic grid, if $p, k, l, m$ are distinct (recall that $n \geq 5$ ), then $u_{p m}=2\left\{u_{p k} u_{k l} u_{m l}\right\}$. However, by (11) and the commutativity of the support projections associated with $u_{i l}$ and $u_{i k}$ (see Lemma 5.4),

$$
\begin{aligned}
u_{p m}= & 2\left\{u_{p k} u_{k l} u_{m l}\right\} \\
= & u_{p k} u_{k l}^{*} u_{m l}+u_{m l} u_{k l}^{*} u_{p k} \\
= & u_{p k}\left(L_{i k} L_{i l} u_{k l}+u_{k l} R_{i l} R_{i k}\right)^{*} u_{m l}+u_{m l}\left(L_{i k} L_{i l} u_{k l}+u_{k l} R_{i l} R_{i k}\right)^{*} u_{n k} \\
= & u_{p k} u_{k l}^{*} L_{i l}\left(L_{i k} u_{m l}\right)+\left(u_{p k} R_{i l}\right) R_{i k} u_{k l}^{*} u_{m l} \\
& +u_{m l} u_{k l}^{*} L_{i k}\left(L_{i l} u_{p k}\right)+\left(u_{m l} R_{i k}\right) R_{i l} u_{k l}^{*} u_{p k}=0,
\end{aligned}
$$

which is a contradiction. Thus $e_{i i} \neq 0$ and it is a partial isometry:

$$
\begin{aligned}
e_{i i} e_{i i}^{*} e_{i i} & =u_{i k} u_{k l}^{*} u_{i l} u_{i l}^{*} u_{k l} u_{i k}^{*}\left(u_{i k} u_{k l}^{*} u_{i l}\right) \\
& =-u_{i k} u_{k l}^{*} u_{i l} u_{i l}^{*}\left(u_{k l} u_{i k}^{*} u_{i l}\right) u_{k l}^{*} u_{i k} \\
& =u_{i k} u_{k l}^{*}\left(u_{i l} u_{i l}^{*} u_{i l}\right) u_{i k}^{*} u_{k l} u_{k l}^{*} u_{i k} \\
& =u_{i k}\left(u_{k l}^{*} u_{i l} u_{i k}^{*}\right) u_{k l} u_{k l}^{*} u_{i k} \\
& =-u_{i k} u_{i k}^{*} u_{i l}\left(u_{k l}^{*} u_{k l} u_{k l}^{*}\right) u_{i k} \\
& =-u_{i k} u_{i k}^{*}\left(u_{i l} u_{k l}^{*} u_{i k}\right) \\
& =u_{i k} u_{i k}^{*} e_{i i} \\
& =u_{i k} u_{i k}^{*}\left(u_{i k} u_{k m}^{*} u_{i m}\right) \\
& =u_{i k} u_{k m}^{*} u_{i m}=e_{i i}
\end{aligned}
$$

Finally, to show orthogonality, take $i, j, l, p$ distinct and note that

$$
e_{i i}^{*} e_{j j}=\left(u_{i l} u_{l k}^{*} u_{k i}\right)^{*} u_{j p} u_{p m}^{*} u_{m j}=u_{k i}^{*} u_{l k} u_{i l}^{*} u_{j p} u_{p m}^{*} u_{m j}=0
$$

and similarly $e_{i i} e_{j j}^{*}=0$.

Lemma 4.2. With the above notation,

(a): $e_{i i} u_{i j}^{*} e_{i i}=e_{i i} e_{j j}^{*} e_{i i}=0$ for $i \neq j$.

(b): $\left\{e_{i i}\right\} \cup\left\{u_{i j}\right\}$ is a linearly independent set.

(c): $u_{i j} \perp e_{k k}$ for $k \notin\{i, j\}$, that $i s, u_{i j} \in A_{0}\left(e_{k k}\right)$.

(d): $\left\{e_{i i} e_{i i} u_{i j}\right\}=u_{i j} / 2=\left\{e_{j j} e_{j j} u_{i j}\right\}$, that $i s, u_{i j} \in A_{1}\left(e_{i i}\right) \cap A_{1}\left(e_{j j}\right)$.

Proof.

$$
\begin{aligned}
e_{i i} u_{i j}^{*} e_{i i} & =u_{i k} u_{k l}^{*} u_{i l} u_{i j}^{*} u_{i k} u_{k l}^{*} u_{i l}=-u_{i l} u_{k l}^{*} u_{i k} u_{i j}^{*} u_{i k} u_{k l}^{*} u_{i l} \\
& =-u_{i l} u_{k l}^{*}\left\{u_{i k} u_{i j} u_{i k}\right\} u_{k l}^{*} u_{i l}=0,
\end{aligned}
$$

since $\left\{u_{i k} u_{i j} u_{i k}\right\} \in A_{2-1+2}\left(u_{i k}\right)=\{0\}$ by Peirce calculus. Also

$$
e_{i i} e_{j j}^{*} e_{i i}=u_{i k} u_{k l}^{*}\left(u_{i l} u_{j p}^{*}\right) u_{p m} u_{j m}^{*} u_{i k} u_{k l}^{*} u_{i l}=0
$$


proving (a), and (b) follows immediately from (a).

To prove (c), note first that $e_{k k}^{*} u_{i j}=\left(u_{k l} u_{l m}^{*} u_{m k}\right)^{*} u_{i j}=u_{m k}^{*} u_{l m} u_{k l}^{*} u_{i j}$ which is zero if $\{i, j\} \cap\{l, k\}=\emptyset$, and similarly $u_{i j} e_{k k}^{*}=0$. Thus $2\left\{u_{i j} u_{i j} e_{k k}\right\}=u_{i j} u_{i j}^{*} e_{k k}+$ $e_{k k} u_{i j}^{*} u_{i j}=0$, which is equivalent to $u_{i j} \in A_{0}\left(e_{k k}\right)$.

Finally, we shall show assertion (d):

$$
\begin{aligned}
2\left\{e_{i i} e_{i i} u_{i j}\right\}= & e_{i i} e_{i i}^{*} u_{i j}+u_{i j} e_{i i}^{*} e_{i i} \\
= & u_{i p} u_{p m}^{*} u_{i m} u_{i l}^{*} u_{k l} u_{i k}^{*} u_{i j}+u_{i j} u_{i k}^{*} u_{l k} u_{i l}^{*} u_{i m} u_{m p}^{*} u_{i p} \\
= & 2 u_{i p}\left\{u_{p m} u_{i m} u_{i l}\right\}^{*} u_{k l} u_{i k}^{*} u_{i j}+2 u_{i j} u_{i k}^{*} u_{l k}\left\{u_{i l} u_{i m} u_{m p}\right\}^{*} u_{i p} \\
& \left(\text { since } u_{p m}^{*} u_{k l}=u_{k l} u_{p m}^{*}=0\right) \\
= & u_{i p} u_{p l}^{*} u_{k l} u_{i k}^{*} u_{i j}-u_{i j} u_{i k}^{*} u_{l k} u_{p l}^{*} u_{i p} \\
= & 2 u_{i p}\left\{u_{p l} u_{k l} u_{i k}\right\}^{*} u_{i j}-2 u_{i j}\left\{u_{i k} u_{l k} u_{p l}\right\}^{*} u_{i p} \\
= & u_{i p}\left(-u_{p i}\right)^{*} u_{i j}+u_{i j} u_{i p}^{*} u_{i p} \\
= & 2\left\{u_{i p} u_{i p} u_{i j}\right\}=u_{i j} .
\end{aligned}
$$

Lemma 4.3. Define, for $i \neq j, e_{i j}=e_{i i} e_{i i}^{*} u_{i j} e_{j j}^{*} e_{j j}$ (product in $\left.A\right)$. Then $u_{i j}=$ $e_{i j}-e_{j i}$ and

(a): $\left\{e_{i j}\right\}$ is a system of matrix units in the $C^{*}$-algebra $A_{2}(v)^{(v)}$.

(b): $\psi$ extends to $a^{*}$-isomorphism $\tilde{\psi}: s p_{\mathbb{C}}\left\{e_{i j}\right\} \rightarrow s p_{\mathbb{C}}\left\{E_{i j}\right\}$ satisfying $\tilde{\psi}\left(e_{i j}\right)=$ $E_{i j}$, where $\left\{E_{i j}\right\}=\phi_{j} \otimes \phi_{i}$.

Proof. By definition, $e_{j i}=e_{j j} e_{j j}^{*} u_{j i} e_{i i}^{*} e_{i i}=-e_{j j} e_{j j}^{*} u_{i j} e_{i i}^{*} e_{i i}=-e_{i j}$, and by Lemmas 4.1 and 4.2 ,

$$
\begin{aligned}
e_{i j}-e_{j i} & =e_{i i} e_{i i}^{*} u_{i j} e_{j j}^{*} e_{j j}+e_{j j} e_{j j}^{*} u_{i j} e_{i i}^{*} e_{i i} \\
& =2\left\{e_{i i} e_{i i} u_{i j}\right\} e_{j j}^{*} e_{j j}+e_{j j} e_{j j}^{*} 2\left\{e_{i i} e_{i i} u_{i j}\right\} \\
& =u_{i j} e_{j j}^{*} e_{j j}+e_{j j} e_{j j}^{*} u_{i j} \\
& =2\left\{e_{j j} e_{j j} u_{i j}\right\}=u_{i j} .
\end{aligned}
$$

Since $v=\sum e_{k k}$, to prove (a) it remains to show that $e_{i j} v^{*} e_{l k}=\delta_{j l} e_{i k}$ and $v e_{i j}^{*} v=$ $e_{j i}$.

In the first place, if $j \neq l$, then $e_{i j} v^{*} e_{l k}=e_{i i} e_{i i}^{*} u_{i j} e_{j j}^{*}\left(e_{j j} v^{*} e_{l l}\right) e_{l l}^{*} u_{l k} e_{k k}^{*} e_{k k}=0$. Now consider the case $j=l$, so that

$$
e_{i j} v^{*} e_{j k}=e_{i i} e_{i i}^{*} u_{i j} e_{j j}^{*} e_{j j}\left(\sum_{q} e_{q q}\right)^{*} e_{j j} e_{j j}^{*} u_{j k} e_{k k}^{*} e_{k k}=e_{i i} e_{i i}^{*} u_{i j} e_{j j}^{*} u_{j k} e_{k k}^{*} e_{k k}
$$

There are five cases to prove:

- $e_{i i} v^{*} e_{i i}=e_{i i}$; this is true since $e_{i i} v^{*} e_{i i}=e_{i i} e_{i i}^{*} e_{i i}=e_{i i}$.

- $e_{i i} v^{*} e_{i k}=e_{i k}, k \neq i$; since $e_{i i} v^{*} e_{i k}=e_{i i} v^{*} e_{i i} e_{i i}^{*} u_{i k} e_{k k}^{*} e_{k k}=e_{i i} e_{i i}^{*} u_{i k} e_{k k}^{*} e_{k k}=$ $e_{i k}$.

- $e_{i j} v^{*} e_{j j}=e_{i j}, i \neq j$; since $e_{i j} v^{*} e_{j j}=e_{i i} e_{i i}^{*} u_{i j} e_{j j}^{*} e_{j j} v^{*} e_{j j}=e_{i j}$. 
- $e_{i j} v^{*} e_{j i}=e_{i i}, i \neq j$; since

$$
\begin{aligned}
e_{i j} v^{*} e_{j i}= & e_{i i} e_{i i}^{*} u_{i j} e_{j j}^{*} e_{j j} v^{*} e_{j j} e_{j j}^{*} u_{j i} e_{i i}^{*} e_{i i} \\
= & e_{i i} e_{i i}^{*} u_{i j} e_{j j}^{*} u_{j i} e_{i i}^{*} e_{i i} \\
= & e_{i i} e_{i i}^{*} u_{i j} u_{j k}^{*} u_{k l} u_{j l}^{*} u_{j i} e_{i i}^{*} e_{i i} \\
= & 2 e_{i i} e_{i i}^{*}\left\{u_{i j} u_{j k} u_{k l}\right\} u_{j l}^{*} u_{j i} e_{i i}^{*} e_{i i} \\
& \left(\text { by Lemma 4.2(c), } e_{i i}^{*} u_{k l}=0\right) \\
= & -e_{i i} e_{i i}^{*} u_{i l} u_{j l}^{*} u_{j i} e_{i i}^{*} e_{i i} \\
= & e_{i i} e_{i i}^{*} e_{i i} e_{i i}^{*} e_{i i}=e_{i i} .
\end{aligned}
$$

- $e_{i j} v^{*} e_{j k}=e_{i k}, i, j, k$ distinct; since

$$
\begin{aligned}
e_{i j} v^{*} e_{j k} & =e_{i i} e_{i i}^{*} u_{i j}\left(e_{j j}^{*} e_{j j} e_{j j}^{*} e_{j j} e_{j j}^{*}\right) u_{j k} e_{k k}^{*} e_{k k} \\
& =e_{i i} e_{i i}^{*} u_{i j} e_{j j}^{*} u_{j k} e_{k k}^{*} e_{k k} \\
& =e_{i i} e_{i i}^{*} u_{i j}\left(u_{j m} u_{m p}^{*} u_{j p}\right)^{*} u_{j k} e_{k k}^{*} e_{k k} \\
& =e_{i i} e_{i i}^{*} u_{i j} u_{j p}^{*} u_{m p} u_{j m}^{*} u_{j k} e_{k k}^{*} e_{k k} \\
& =2 e_{i i} e_{i i}^{*}\left\{u_{i j} u_{j p} u_{m p}\right\} u_{j m}^{*} u_{j k} e_{k k}^{*} e_{k k} \\
& =e_{i i} e_{i i}^{*} u_{i m} u_{j m}^{*} u_{j k} e_{k k}^{*} e_{k k} \\
& =2 e_{i i} e_{i i}^{*}\left\{u_{i m} u_{j m} u_{j k}\right\} e_{k k}^{*} e_{k k} \\
& =e_{i i} e_{i i}^{*} u_{i k} e_{k k}^{*} e_{k k}=e_{i k} .
\end{aligned}
$$

Finally,

$$
\begin{aligned}
e_{i j} & =e_{i i} e_{i i}^{*} u_{i j} e_{j j}^{*} e_{j j} \\
& =u_{i k} u_{k l}^{*} u_{i l} u_{i l}^{*} u_{k l}\left(u_{i k}^{*} u_{i j} u_{j m}^{*}\right) u_{p m} u_{j p}^{*} u_{j p} u_{p m}^{*} u_{j m} \\
& =2 u_{i k} u_{k l}^{*} u_{i l} u_{i l}^{*} u_{k l}\left\{u_{i k} u_{i j} u_{j m}\right\}^{*} u_{p m} u_{j p}^{*} u_{j p} u_{p m}^{*} u_{j m} \\
& =-u_{i k} u_{k l}^{*} u_{i l} u_{i l}^{*} u_{k l}\left(u_{m k}^{*} u_{p m} u_{j p}^{*}\right) u_{j p} u_{p m}^{*} u_{j m} \\
& =u_{i k} u_{k l}^{*} u_{i l}\left(u_{i l}^{*} u_{k l} u_{j k}^{*}\right) u_{j p} u_{p m}^{*} u_{j m} \\
& =-u_{i k} u_{k l}^{*} u_{i l} u_{i j}^{*} u_{j p} u_{p m}^{*} u_{j m} \\
& =-e_{i i} u_{i j}^{*} e_{j j}
\end{aligned}
$$

and therefore $v e_{i j}^{*} v=-v e_{j j}^{*} u_{i j} e_{i i}^{*} v=-e_{j j} e_{j j}^{*} u_{i j} e_{i i}^{*} e_{i i}=e_{j j} e_{j j}^{*} u_{j i} e_{i i}^{*} e_{i i}=e_{j i}$. This completes the proof of (a).

Since the system of matrix units $\left\{e_{i j}\right\}$ are linearly independent, $\tilde{\psi}$ defines a ${ }^{*}$-isomorphism of $\operatorname{sp}_{\mathbb{C}}\left\{e_{i j}\right\}$ onto $\mathrm{sp}_{\mathbb{C}}\left\{E_{i j}\right\}$, proving (b).

By the same method used in Section 3 for the Type 3 case, $\tilde{\psi}$ extends to a *-isomorphism of $\overline{\operatorname{sp}_{\mathbb{C}}\left\{e_{i j}\right\}}{ }^{*}$ onto $B(H)$. The proof of Proposition 2.6 and Theorem 3 is thus complete in the case that $Y$ is triple isomorphic to a Cartan factor of type 2 .

\section{Cartan factors of type 1}

In this and the next section, we prove Proposition 2.6 and Theorem 3 in the case that $Y$ is triple isomorphic to a Cartan factor of type 1. This turns out to be more complicated than the other types, especially in the case that $Y$ is of rank 1 (Hilbertian). Except for some important preliminary cases (see subsection 5.3), the rank 1 case is proved in section 7 . 
Let $\left\{u_{i j}: i \in \Lambda, j \in \Sigma\right\}$ be a rectangular grid which is $\mathrm{w}^{*}$-total in $Y$. Recall $(\varangle$, p. 313] that this means that each $u_{i j}$ is a minimal partial isometry, $u_{j k} \perp u_{i l}$ if $i \neq j$ and $k \neq l ; u_{j k} \top u_{i l}$ if either $j=i, k \neq l$ or $j \neq i, k=l$;

$$
\left\{u_{j k} u_{j l} u_{i l}\right\}=u_{i k} / 2 \text { if } j \neq i \text { and } k \neq l \text {; }
$$

and all other triple products are zero.

We shall assume throughout this section that $Y$ is triple isomorphic to $B(H, K)$, that is, $|\Lambda|=\operatorname{dim} K$ and $|\Sigma|=\operatorname{dim} H$. Specifically, by [8, p.317 and Lemma 1.14], this means that the map $u_{i j} \mapsto E_{i j}$ extends to a triple isomorphism of $Y$ onto $B(H, K)$, where $E_{i j}=\phi_{i} \otimes \psi_{j}$ for orthonormal bases $\left\{\psi_{j}: j \in \Sigma\right\}$ in $H$ and $\left\{\phi_{i}: i \in \Lambda\right\}$ in $K$.

5.1. A special case. Note that the canonical rectangular grid $\left\{E_{i j}\right\}$ for $B(H, K)$ satisfies $E_{i j} E_{i k}^{*}=\left(\psi_{k} \mid \psi_{j}\right) \phi_{i} \otimes \phi_{i}=0$ for $j \neq k$ and all $i$; and $E_{i k}^{*} E_{j k}=0$ for $i \neq j$ and all $k$.

Lemma 5.1. With $Y$ as above, assume that for some fixed values of $i \in \Lambda, k, l \in \Sigma$, $u_{i l} u_{i k}^{*}=0$ and $k \neq l$, or for some fixed values of $i, j \in \Lambda, k \in \Sigma, u_{i k}^{*} u_{j k}=0$ and $i \neq j$. Then

(a): for all $j \in \Lambda, p, q \in \Sigma$ with $p \neq q, u_{j p} u_{j q}^{*}=0$ and for all $p, q \in \Lambda, r \in \Sigma$ with $p \neq q, u_{p r}^{*} u_{q r}=0$.

(b): $Y$ is a ternary subtriple of $A$ which is ternary isomorphic and completely isometric to $B(H, K)$.

Proof. We shall give the proof in the case that $u_{i l} u_{i k}^{*}=0$. The other case follows by symmetry.

We first take care of the " $i^{\text {th }}$-row," where $i, k, l$ are the fixed values. If $p \notin\{k, l\}$,

$$
\begin{aligned}
u_{i p} u_{i k}^{*} & =2\left\{u_{i l} u_{i l} u_{i p}\right\} u_{i k}^{*} \\
& =\left(u_{i l} u_{i l}^{*} u_{i p}+u_{i p} u_{i l}^{*} u_{i l}\right) u_{i k}^{*} \\
& =u_{i l}\left(u_{i l}^{*} u_{i p} u_{i k}^{*}\right)(\text { by assumption }) \\
& =-u_{i l} u_{i k}^{*} u_{i p} u_{i l}^{*}\left(\text { since }\left\{u_{i k} u_{i p} u_{i l}\right\}=0\right) \\
& =0 .
\end{aligned}
$$

Thus, if $q \notin\{p, k\}$,

$$
\begin{aligned}
u_{i p} u_{i q}^{*} & =2 u_{i p}\left\{u_{i k} u_{i k} u_{i q}\right\}^{*} \\
& =u_{i p}\left(u_{i k} u_{i k}^{*} u_{i q}+u_{i q} u_{i k}^{*} u_{i k}\right)^{*} \\
& =\left(u_{i p} u_{i q}^{*} u_{i k}\right) u_{i k}^{*}(\operatorname{by}(13)) \\
& =-u_{i k} u_{i q}^{*} u_{i p} u_{i k}^{*}=0 .
\end{aligned}
$$

This proves the first statement in (a) when $j$ has the value $i$. We next take care of the " $j$ th-row" (if it exists). If $p \neq q$ and $j \neq i$,

$$
\begin{aligned}
u_{j p} u_{j q}^{*} & =2\left\{u_{i p} u_{i q} u_{j q}\right\} u_{j q}^{*} \\
& =\left(u_{i p} u_{i q}^{*} u_{j q}+u_{j q} u_{i q}^{*} u_{i p}\right) u_{j q}^{*} \\
& =u_{j q} u_{i q}^{*} u_{i p} u_{j q}^{*}(\mathrm{by}(14)) \\
& =2 u_{j q} u_{i q}^{*} u_{i p}\left\{u_{i q} u_{i p} u_{j p}\right\}^{*} \\
& =u_{j q} u_{i q}^{*} u_{i p}\left(u_{i q}^{*} u_{i p} u_{j p}^{*}+u_{j p}^{*} u_{i p} u_{i q}^{*}\right) \\
& \left.=0 \text { (by (14) and the minimality of } u_{i p}\right) .
\end{aligned}
$$


This proves the first statement in (a). We complete the proof of (a), by taking care of the "columns," (if they exist). For all $p, q, r$ with $p \neq q$, choose $s \neq r$. Then

$$
\begin{aligned}
u_{p r}^{*} u_{q r} & =2 u_{p r}^{*}\left\{u_{q s} u_{p s} u_{p r}\right\}=u_{p r}^{*}\left(u_{p r} u_{p s}^{*} u_{q s}+u_{q s} u_{p s}^{*} u_{p r}\right) \\
& =u_{p r}^{*}\left(u_{p r} u_{p s}^{*}\right) u_{q s}+\left(u_{p r}^{*} u_{q s}\right) u_{p s}^{*} u_{p r}=0,
\end{aligned}
$$

by orthogonality of $u_{q s}$ and $u_{p r}$ for $s \neq r$ and by (15).

By (a), and the separate $\mathrm{w}^{*}$-continuity of multiplication, $Y$ is a ternary subtriple (closed under $\left.(a, b, c) \mapsto a b^{*} c\right)$. Furthermore, $\operatorname{sp}_{\mathbb{C}}\left\{u_{i j}\right\}$ is ternary isomorphic and isometric to $\operatorname{sp}_{\mathbb{C}}\left\{E_{i j}\right\}$ via $u_{i j} \mapsto E_{i j}$ and we can again use [8, Lemma 1.14] to extend the map to a ternary isomorphism of $Y$ onto $B(H, K)$, which then is a complete isometry.

By the same arguments, we also have the following.

Lemma 5.2. With $Y$ as above, assume that for some fixed values of $i, k, l, u_{i l}^{*} u_{i k}=$ 0 and $k \neq l$, or $u_{i k} u_{j k}^{*}=0$ and $i \neq j$. Then

(a): for all $i, k, l$ with $k \neq l, u_{i l}^{*} u_{i k}=0$ and for all $i, j, k$ with $i \neq j, u_{i k} u_{j k}^{*}=0$.

(b): $Y$ is ternary isomorphic and completely isometric to $B(K, H)$.

It is convenient to single out the rank one case.

Corollary 5.3. Let $Y$ be triple isomorphic to a Cartan factor of type 1 and rank 1 and denote by $\left\{u_{\lambda}\right\}$ a rank 1 rectangular grid for $Y$.

(a): If $u_{i} u_{j}^{*}=0$ for some $i \neq j$, then $Y$ is completely isometric to $B(H, \mathbb{C})$.

(b): If $u_{i}^{*} u_{j}=0$ for some $i \neq j$, then $Y$ is completely isometric to $B(\mathbb{C}, K)$.

5.2. The case of rank 2 or more. The following simple lemma will be useful in this and the next section. Part (b) of it is referred to as "hopping".

Lemma 5.4. Let $u, v, w$ be partial isometries.

(a): If $u$ and $w$ are colinear, then the support projections $u u^{*}, w w^{*}$ commute as do $u^{*} u, w^{*} w$.

(b): If $v$ and $w$ are each colinear with $u$, then $u u^{*} v w^{*}=v w^{*} u u^{*}$ and $u^{*} u v^{*} w=$ $v^{*} w u^{*} u$.

Proof. We prove (b) first. Since $u u^{*} v+v u^{*} u=v$ and $u u^{*} w+w u^{*} u=w$, $\left(u u^{*} v\right) w^{*}=\left(v-v u^{*} u\right) w^{*}=v w^{*}-v\left(u^{*} u w^{*}\right)=v w^{*}-v\left(w^{*}-w^{*} u u^{*}\right)=v w^{*} u u^{*}$. Similarly for the second statement. To prove (a) use the same argument: $u u^{*} w w^{*}=$ $\left(w-w u^{*} u\right) w^{*}=w w^{*}-w\left(u^{*} u w^{*}\right)=w w^{*}-w\left(w^{*}-w^{*} u u^{*}\right)=w w^{*} u u^{*}$.

Justified by Lemmas 5.1 and 5.2 we may now assume in the rest of this subsection 5.2, without loss of generality, that

$$
u_{i k} u_{i j}^{*} \neq 0 \text { and } u_{i k}^{*} u_{i j} \neq 0 \text { for all } i \in \Lambda, j, k \in \Sigma
$$

$$
u_{i k} u_{j k}^{*} \neq 0 \text { and } u_{i k}^{*} u_{j k} \neq 0 \text { for all } i, j \in \Lambda, k \in \Sigma \text {. }
$$

Lemma 5.5. Suppose that $Y$ is triple isomorphic to a Cartan factor $B(H, K)$ of type 1 and rank at least 2 , let $\left\{u_{i j}: i \in \Lambda, j \in \Sigma\right\}$ be a rectangular grid for $Y$ and suppose that (16) and (17) hold.

Then for all $i \in \Lambda$ and $j \in \Sigma$, the projections

$$
L_{i}:=\prod_{k \in \Sigma} u_{i k} u_{i k}^{*} \text { and } R_{j}:=\prod_{l \in \Lambda} u_{l j}^{*} u_{l j}
$$


are non-zero.

Proof. Note that by Lemma 5.4, the above are products of commuting projections. We shall show that $L_{i} \neq 0$, the proof for $R_{j}$ being similar.

Suppose the assertion is false, that is, for some $i \in \Lambda$,

$$
\prod_{k \in \Sigma} u_{i k} u_{i k}^{*}=0
$$

Choose a finite subset $S \subseteq \Sigma$ and denote it by $\{1,2, \cdots n\}$. Choose a $j \neq i$ and an $l \in S-\{1\}$. Since $u_{i l} \top u_{i 1}$,

$$
\begin{aligned}
u_{i l} u_{j l}^{*} & =u_{i 1} u_{i 1}^{*} u_{i l} u_{j l}^{*}+u_{i l} u_{i 1}^{*} u_{i 1} u_{j l}^{*} \\
& =u_{i 1} u_{i 1}^{*} u_{i l} u_{j l}^{*} \\
& =u_{i 1} u_{i 1}^{*} u_{i 2} u_{i 2}^{*} u_{i l} u_{j l}^{*} \\
& =\cdots \\
& =\left(\prod_{k=1, k \neq l}^{n} u_{i k} u_{i k}^{*}\right) u_{i l} u_{j l}^{*} \\
& =\left(\prod_{k=1}^{n} u_{i k} u_{i k}^{*}\right) u_{i l} u_{j l}^{*}
\end{aligned}
$$

Since $\prod_{k \in \Sigma} u_{i k} u_{i k}^{*}$ is the $\mathrm{w}^{*}$-limit of the net $\left\{\prod_{k \in S} u_{i k} u_{i k}^{*}\right\}_{|S|<\infty}$, it follows by separate $\mathrm{w}^{*}$-continuity of multiplication that $u_{i l} u_{j l}^{*}=0$, which contradicts (17).

Lemma 5.6. Let $Y$ be as in Lemma5.9. Let

$$
p=\sum_{i \in \Lambda} \prod_{k \in \Sigma} u_{i k} u_{i k}^{*}
$$

which is a sum of non-zero orthogonal projections. The maps $Y \ni y \mapsto p y \in p Y$ and $Y \ni y \mapsto(1-p) y \in(1-p) Y$ are completely contractive triple isomorphisms. Also, $p Y \perp(1-p) Y$.

Proof. We begin by showing that $\left\{p u_{i j}\right\}$ is a rectangular grid which is $\mathrm{w}^{*}$-total in its $\mathrm{w}^{*}$-closure. We start by showing that $p u_{i j}$ is a minimal partial isometry using the criterion (7). We have

$$
p u_{i j}\left(p u_{k l}\right)^{*} p u_{i j}=p u_{i j} u_{k l}^{*} p u_{i j}=p u_{i j} u_{k l}^{*}\left(\sum_{q \in \Lambda} u_{q 1} u_{q 1}^{*} \cdots u_{q n} u_{q n}^{*}\right) u_{i j} .
$$

By Lemma 5.4(a), this is zero if $k \neq i$. For $k=i$, we have

$$
p u_{i j}\left(p u_{k l}\right)^{*} p u_{i j}=p u_{i j} u_{i l}^{*} u_{i 1} u_{i 1}^{*} \cdots u_{i n} u_{i n}^{*} u_{i j}
$$

which is zero for $j \neq l$, since by Peirce calculus, $u_{i j} u_{i l}^{*} u_{i j}=0$. On the other hand

$$
\left(p u_{i j}\right)\left(p u_{i j}\right)^{*}\left(p u_{i j}\right)=p u_{i j} u_{i j}^{*} u_{i 1} u_{i 1}^{*} \cdots u_{i n} u_{i n}^{*} u_{i j}=p u_{i j}
$$

and it is non-zero by Lemma 5.5. This proves that $p u_{i j}$ is a minimal partial isometry.

We next show that $p u_{j k} \perp p u_{i l}$ for $i \neq j$ and $k \neq l$. On the one hand, $p u_{j k}\left(p u_{i l}\right)^{*}=p u_{j k} u_{i l}^{*} p=0$; and on the other hand,

$$
\left(p u_{i l}\right)^{*} p u_{j k}=u_{i l}^{*} p u_{j k}=\sum_{q \in \Lambda} u_{i l}^{*}\left(u_{q 1} u_{q 1}^{*} \cdots u_{q n} u_{q n}^{*}\right) u_{j k}=u_{i l}^{*} u_{i 1} u_{i 1}^{*} \cdots u_{i n} u_{i n}^{*} u_{j k}=0
$$


by Lemma 5.4(a).

We next show that $p u_{i k} \top p u_{i l}$ for $k \neq l$. We have

$$
\begin{aligned}
p u_{i k}\left(p u_{i k}\right)^{*} p u_{i l}+p u_{i l}\left(p u_{i k}\right)^{*} p u_{i k}= & \\
& p u_{i k} u_{i k}^{*} p u_{i l}+p u_{i l} u_{i k}^{*} p u_{i k} \\
= & \left(u_{i 1} u_{i 1}^{*} \cdots u_{i n} u_{i n}^{*}\right) u_{i k} u_{i k}^{*} p u_{i l} \\
+ & \left(u_{i 1} u_{i 1}^{*} \cdots u_{i n} u_{i n}^{*}\right) u_{i l} u_{i k}^{*} p u_{i k} \\
= & \left(u_{i 1} u_{i 1}^{*} \cdots u_{i n} u_{i n}^{*}\right) u_{i l}+0\left(\text { since } u_{i k}^{*} u_{i l} u_{i k}^{*}=0\right) \\
= & p u_{i l} \text { as required }
\end{aligned}
$$

We next show that for $i \neq j, p u_{j k} \top p u_{i k}$. To this end, we shall show that $p u_{j k}\left(p u_{j k}\right)^{*} p u_{i k}=0$ and $p u_{i k}\left(p u_{j k}\right)^{*} p u_{j k}=p u_{i k}$. In the first place,

$$
p u_{j k}\left(p u_{j k}\right)^{*} p u_{i k}=\left(p u_{j k} u_{j k}^{*}\right)\left(p u_{i k}\right)=\left(u_{j 1} u_{j 1}^{*} \cdots u_{j n} u_{j n}^{*}\right)\left(u_{i 1} u_{i 1}^{*} \cdots u_{i n} u_{i n}^{*}\right)=0
$$

In the second place,

$$
\begin{aligned}
p u_{i k}\left(p u_{j k}\right)^{*} p u_{j k}= & p u_{i k} u_{j k}^{*}\left(p u_{j k}\right) \\
= & p u_{i k} u_{j k}^{*}\left(\prod_{1 \leq l \leq n, l \neq k} u_{j l} u_{j l}^{*}\right) u_{j k} \\
= & p u_{i k}\left(u_{j k}^{*}-u_{j 1}^{*} u_{j 1} u_{j k}^{*}\right) u_{j 2} u_{j 2}^{*} \cdots u_{j n} u_{j n}^{*} u_{j k} \\
= & p u_{i k} u_{j k}^{*}\left(u_{j 2} u_{j 2}^{*} \cdots u_{j n} u_{j n}^{*}\right) u_{i k} \\
& \cdots \\
= & p u_{i k} u_{j k}^{*} u_{j k}=p\left(u_{i k}-u_{j k} u_{j k}^{*} u_{i k}\right)=p u_{i k}
\end{aligned}
$$

Finally we shall show that $\left\{p u_{j k}, p u_{j l}, p u_{i l}\right\}=p u_{i k} / 2$ for $j \neq i$ and $l \neq k$. It suffices to prove $p u_{j k} u_{j l}^{*} p u_{i l}=0$ and $p u_{i l} u_{j l}^{*} p u_{j k}=p u_{i k}$.

On the one hand,

$$
\begin{aligned}
p u_{j k} u_{j l}^{*} p u_{i l} & =\sum_{m \in \Lambda} u_{m 1} u_{m 1}^{*} \cdots u_{m n} u_{m n}^{*} u_{j k} u_{j l}^{*} p u_{i l} \\
& =u_{j 1} u_{j 1}^{*} \cdots u_{j n} u_{j n}^{*} u_{j k} u_{j l}^{*} p u_{i l}=0,
\end{aligned}
$$

since $u_{j l}^{*} u_{j k} u_{j l}^{*}=0$. 
On the other hand,

$$
\begin{aligned}
p u_{i l} u_{j l}^{*} p u_{j k}= & \sum_{m \in \Lambda} p u_{i l} u_{j l}^{*} u_{m 1} u_{m 1}^{*} \cdots u_{m n} u_{m n}^{*} u_{j k} \\
= & p u_{i l} u_{j l}^{*}\left[u_{j 1} u_{j 1}^{*} \cdots u_{j n} u_{j n}^{*}\right] u_{j k} \\
& \left(\text { where } u_{j l} u_{j l}^{*} \text { and } u_{j k} u_{j k}^{*} \text { are not present in the }[\cdot]\right) \\
= & p u_{i l}\left[u_{j l}^{*}-u_{j 1}^{*} u_{j 1} u_{j l}^{*}\right] u_{j 2} u_{j 2}^{*} \cdots u_{j n} u_{j n}^{*} u_{j k} \\
= & p u_{i l} u_{j l}^{*} u_{j 2} u_{j 2}^{*} \cdots u_{j n} u_{j n}^{*} u_{j k} \\
= & \cdots \\
= & p u_{i l} u_{j l}^{*} u_{j k} \\
= & p\left(u_{i k}-u_{j k} u_{j l}^{*} u_{i l}\right) \\
= & p u_{i k}-p u_{j k} u_{j l}^{*} u_{i l} \\
= & p u_{i k}-\sum_{m \in \Lambda} u_{m 1} u_{m 1}^{*} \cdots u_{m n} u_{m n}^{*} u_{j k} u_{j l}^{*} u_{i l} \\
= & p u_{i k}-u_{j 1} u_{j 1}^{*} \cdots u_{j n} u_{j n}^{*} u_{j k} u_{j l}^{*} u_{i l} \\
= & p u_{i k} \operatorname{since} u_{j l}^{*} u_{j k} u_{j l}^{*}=0 .
\end{aligned}
$$

It now follows that the map $y \mapsto p y$ is a triple isomorphism, and hence an isometry, from the norm closure $U$ of $\operatorname{sp}_{\mathbb{C}}\left\{u_{i j}\right\}$ onto the norm closure $V$ of $\operatorname{sp}_{\mathbb{C}}\left\{p u_{i j}\right\}$.

We claim that the map $y \mapsto p y$ is an isometry of the $\mathrm{w}^{*}$-closure $\bar{U}$ of $U$ onto the w $^{*}$-closure $\bar{V}$ of $V$, and is thus a complete contraction as well.

First we show that if $p y=0$ and $y \in Y$, then $y=0$, from which it follows that the map $y \mapsto p y$ is a $\mathrm{w}^{*}$-homeomorphism when restricted to the unit ball of $U$. Then by 19 , (3.1)], $y \mapsto p y$ extends to an isometry of $\bar{U}$ onto $\bar{V}$, which is $\mathrm{w}^{*}$-continuous by the uniqueness of the preduals. This $\mathrm{w}^{*}$-extension must agree with $y \mapsto p y$ on $Y$, which proves the claim.

To prove the above statement suppose $p y=0$ for some $y \in Y$. Then $L_{i} y=0$ for each $i \in \Lambda$. We may write $y=\sum \lambda_{i j} u_{i j}$ where the sum converges in the $\mathrm{w}^{*}$-topology and $\lambda_{i j}=l_{i j} y r_{i j}$, where $l_{i j}=u_{i j} u_{i j}^{*}$, (resp. $r_{i j}=u_{i j}^{*} u_{i j}$ ) is the left (resp. right) support of $u_{i j}$. Since $L_{i} u_{i j} R_{j}=L_{i} u_{i j} \neq 0,0=L_{i} y R_{j}=L_{i} l_{i j} y r_{i j} R_{j}=\lambda_{i j} L_{i} u_{i j} R_{j}$ and so $\lambda_{i j}=0$ and $y=0$.

We can similarly show that $\left\{(1-p) u_{i j}\right\}$ is a rectangular grid and that hence, as above, the map $Y \ni y \mapsto(1-p) y \in(1-p) Y$ is an isometry and complete contraction. For example, to prove that $(1-p) u_{j k} \top(1-p) u_{i k}$, it suffices to show that

$$
(1-p) u_{j k}\left[(1-p) u_{i k}\right]^{*}(1-p) u_{i k}=0
$$

and

$$
(1-p) u_{i k}\left[(1-p) u_{i k}\right]^{*}(1-p) u_{j k}=(1-p) u_{j k} .
$$

For the first statement,

$$
\begin{aligned}
& (1-p) u_{j k} u_{i k}^{*}(1-p) u_{i k}=(1-p) u_{j k} u_{i k}^{*} u_{i k}-(1-p) u_{j k} u_{i k}^{*} p u_{i k} \\
& \quad=(1-p) u_{j k} u_{i k}^{*} u_{i k}-(1-p) u_{j k} u_{i k}^{*}\left(u_{i 1} u_{i 1}^{*} \cdots u_{i n} u_{i n}^{*}\right) u_{i k} \\
& \quad=(1-p) u_{j k} u_{i k}^{*} u_{i k}-(1-p) u_{j k} u_{i k}^{*} u_{i k}=0,
\end{aligned}
$$

since in the second term, for $l \neq k, u_{j k} u_{i k}^{*} u_{i l} u_{i l}^{*}=u_{j k}\left(u_{i k}^{*}-u_{i l}^{*} u_{i l} u_{i k}^{*}\right)=u_{j k} u_{i k}^{*}$. 
For the second statement,

$$
\begin{aligned}
(1-p) u_{i k} u_{i k}^{*}(1-p) u_{j k} & =(1-p) u_{i k} u_{i k}^{*} u_{j k}-(1-p) u_{i k} u_{i k}^{*} p u_{j k} \\
& =(1-p) u_{i k} u_{i k}^{*} u_{j k}+0 \\
& =(1-p)\left(u_{j k}-u_{j k} u_{i k}^{*} u_{i k}\right)=(1-p) u_{j k}
\end{aligned}
$$

We omit the entirely analogous calculations showing that the other grid properties hold.

As above, the fact that $y \mapsto(1-p) y$ is a complete semi-isometry follows from the fact that it is one-to-one on $Y$. To see that it is one-to-one on $Y$, suppose $(1-p) y=0$ for some $y \in Y$. Writing $y=\sum \lambda_{i j} u_{i j}$ leads to $\sum_{i, j} \lambda_{i j}\left(1-L_{i}\right) u_{i, j}=0$. If there were indices $(i, j)$ such that $\left(1-L_{i}\right) u_{i, j}=0$, then since $\left(1-L_{i}\right) u_{i, j}=(1-p) u_{i j}$, we would have $u_{k j}^{*} u_{i j}=u_{k j}^{*} p u_{i j}=u_{k j}^{*} L_{i} u_{i j}=0$ for some $k$, violating (17).

Finally we show that $p Y \perp(1-p) Y$. It suffices to show that basis elements are orthogonal. First,

$$
p u_{i j}\left[(1-p) u_{i j}\right]^{*}=p u_{i j} u_{i j}^{*}(1-p)=\left(\prod_{k} u_{i k} u_{i k}^{*}\right) u_{i j} u_{i j}^{*}(1-p)=p(1-p)=0 .
$$

Next, if $j \neq k, p u_{i j} u_{i k}^{*}(1-p)=\left(\prod_{l} u_{i l} u_{i l}^{*}\right) u_{i j} u_{i k}^{*}(1-p)=0$, since $u_{i l} u_{i j}^{*} u_{i l}=0$. Finally, if $k \neq i$,

$$
\begin{aligned}
p u_{i j} u_{k j}^{*}(1-p)= & p u_{i j} u_{k j}^{*}-p u_{i j} u_{k j}^{*} \prod_{l=1, l \neq j}^{n} u_{k l} u_{k l}^{*} \\
= & p u_{i j} u_{k j}^{*}-p u_{i j}\left[u_{k j}-u_{k 1} u_{k 1}^{*} u_{k j}\right]^{*} \prod_{l=2, l \neq j}^{n} u_{k l} u_{k l}^{*} \\
= & p u_{i j} u_{k j}^{*}-p u_{i j} u_{k j}^{*} \prod_{l=2, l \neq j}^{n} u_{k l} u_{k l}^{*} \\
& \ldots \\
= & p u_{i j} u_{k j}^{*}-p u_{i j} u_{k j}^{*}=0 .
\end{aligned}
$$

Clearly $[(1-p) y]^{*} p z=0$ for all $y, z \in Y$, finishing the proof.

Proposition 5.7. Suppose that $Y$ is triple isomorphic to $B(H, K)$ and is of rank at least 2, and that (16) and (17) hold. Then $Y$ is completely semi-isometric to $B(H, K)$ and completely isometric to

$$
\operatorname{Diag}(B(H, K), B(K, H))
$$

Proof. For $k \neq j$,

$$
\begin{aligned}
p u_{i k}\left(p u_{i j}\right)^{*} & =p u_{i k} u_{i j}^{*} p \\
& =p u_{i k} u_{i j}^{*} u_{i 1} u_{i 1}^{*} \cdots u_{i n} u_{i n}^{*}=0 .
\end{aligned}
$$

so Lemma 5.1 applies to show that $p Y$ is completely isometric to $B(H, K)$. By Lemma 5.6, $Y$ is completely semi-isometric to $B(H, K)$. 
Similarly, Lemma 5.1 applies to show that $(1-p) Y$ is completely isometric to $B(K, H)$. Indeed,

$$
\begin{aligned}
{\left[(1-p) u_{i k}\right]\left[(1-p) u_{j k}\right]^{*} } & =(1-p) u_{i k} u_{j k}^{*}(1-p) \\
& =\left(1-\prod_{l} u_{i l} u_{i l}^{*}\right) u_{i k} u_{j k}^{*}(1-p) \\
& =u_{i k} u_{j k}^{*}(1-p)-\left(\prod_{l} u_{i l} u_{i l}^{*}\right) u_{i k} u_{j k}^{*}(1-p) \\
& =u_{i k} u_{j k}^{*}(1-p)-u_{i k} u_{j k}^{*}(1-p)=0,
\end{aligned}
$$

since for $l \neq k, u_{i l} u_{i l}^{*} u_{i k} u_{j k}^{*}=\left(u_{i k}-u_{i k} u_{i l}^{*} u_{i l}\right) u_{j k}^{*}=u_{i k} u_{j k}^{*}$.

As in the proof of Lemma 2.4. $p Y \oplus^{\ell^{\infty}}(1-p) Y$ is completely isometric to $B(H, K) \oplus^{\ell^{\infty}} B(K, H)$.

This completes the proof of Proposition 2.6 and Theorem 3 in the case that $Y$ is of rank 2 or more and triple isomorphic to $B(H, K)$.

5.3. The case of rank 1. Preliminary cases. Assume now that $Y$ is finite dimensional and rank 1. Let us denote a finite rectangular grid of rank 1 for $Y$ by $\left\{u_{1}, \ldots, u_{n}\right\}$. For the record, let us note that this means precisely that $u_{i}$ is a non-zero partial isometry: $\left\{u_{i} u_{i} u_{i}\right\}=u_{i} \neq 0 ; u_{i}$ is minimal:

$$
\left\{u_{i} u_{j} u_{i}\right\}=0 \text { for } i \neq j \text {; }
$$

and that $u_{i}$ is colinear with $u_{k}:\left\{u_{i} u_{i} u_{k}\right\}=u_{k} / 2$ for $i \neq k$. By the grid properties and the identity $\left\|y y^{*} y\right\|=\|y\|^{3}, Y$ is isometric to a Hilbert space with orthonormal basis $\left\{u_{j}\right\}$ (see [8, p.306]).

We shall denote, for $J=\left\{j_{1}, \ldots, j_{i}\right\} \subset\{1,2, \ldots, n\}, u_{j_{1}}^{*} u_{j_{1}} u_{j_{2}}^{*} u_{j_{2}} \cdots u_{j_{i}}^{*} u_{j_{i}}$ by $\left(u^{*} u\right)_{J}$. By commutativity of the projections $u_{k}^{*} u_{k}$ we may and shall assume that $1 \leq j_{1}<\cdots<j_{i} \leq n$. Similarly $\left(u u^{*}\right)_{J}$ will denote $u_{j_{1}} u_{j_{1}}^{*} u_{j_{2}} u_{j_{2}}^{*} \cdots u_{j_{i}} u_{j_{i}}^{*}$.

Lemma 5.8. If $\left(u u^{*}\right)_{J}=0$ for some $J$ with $|J|=i$, then $\left(u u^{*}\right)_{J}=0$ for all $J$ with $|J|=i$. If $\left(u^{*} u\right)_{J}=0$ for some $J$ with $|J|=i$, then $\left(u^{*} u\right)_{J}=0$ for all $J$ with $|J|=i$.

Proof. Suppose that $\left(u u^{*}\right)_{J}=0$ for some $J$ with $|J|=i$. Then for $s \in J$ and $k \notin J$,

$$
\begin{aligned}
\left(u u^{*}\right)_{(J-\{s\}) \cup\{k\}} & =\left(u u^{*}\right)_{J-\{s\}} u_{k} u_{k}^{*} \\
& =\left(u u^{*}\right)_{J-\{s\}}\left(u_{k} u_{s}^{*} u_{s}+u_{s} u_{s}^{*} u_{k}\right) u_{k}^{*} \\
& =\left(u u^{*}\right)_{J-\{s\}} u_{k} u_{s}^{*} u_{s} u_{k}^{*} \\
& =u_{k} u_{s}^{*}\left(u u^{*}\right)_{J-\{s\}} u_{s} u_{k}^{*} \text { (by Lemma 5.4) } \\
& =u_{k} u_{s}^{*} u_{s} u_{s}^{*}\left(u u^{*}\right)_{J-\{s\}} u_{s} u_{k}^{*} \\
& =u_{k} u_{s}^{*}\left(u u^{*}\right)_{J} u_{s} u_{k}^{*}=0 .
\end{aligned}
$$

The proof of the second statement is similar.

Lemma 5.8 makes it possible to define $i_{R}$ to be the largest $i$ such that $\left(u u^{*}\right)_{J} \neq 0$ for any $J$ with $|J|=i$ and $i_{L}$ to be the largest $i$ such that $\left(u^{*} u\right)_{J} \neq 0$ for any $J$ with $|J|=i$. The numbers $i_{R}$ and $i_{L}$ are indices which depend on how a $J C^{*}$-triple sits in its ternary envelope. We use the numbers $i_{R}$ and $i_{L}$ to define projections $p_{R}=\sum_{|J|=i_{R}}\left(u u^{*}\right)_{J}$ and $p_{L}=\sum_{|J|=i_{L}}\left(u^{*} u\right)_{J}$. 
Lemma 5.9. Each of the maps $y \mapsto p_{R} y, y \mapsto y p_{L}, y \mapsto\left(1-p_{R}\right) y, y \mapsto y\left(1-p_{L}\right)$ are completely contractive triple isomorphisms of $Y$ into A. Also $p_{R} Y \perp\left(1-p_{R}\right) Y$ and $Y p_{L} \perp Y\left(1-p_{L}\right)$.

Proof. To prove the first statement, it suffices to show that each of these maps takes the rectangular rank 1 grid $\left\{u_{k}\right\}_{k=1}^{n}$ into a rectangular grid of rank 1 .

We carry out the proof for $y \mapsto p_{R} y$ and $y \mapsto\left(1-p_{R}\right) y$, the proofs for the other maps being identical. For notation's sake, we let $p=p_{R}$ and $w_{j}=p u_{j}$.

If $w_{k}=0$, then

$$
0=w_{k} w_{k}^{*}=\left(p u_{k}\right)\left(p u_{k}\right)^{*}=p u_{k} u_{k}^{*} p=\sum_{|J|=i_{R}, k \in J}\left(u u^{*}\right)_{J} .
$$

A sum of orthogonal projections cannot be zero unless each one is. Thus $\left(u u^{*}\right)_{J}=0$ for any $J$ containing $k$ with $|J|=i_{R}$, which is a contradiction. Hence, $w_{k} \neq 0$.

Next, for $i \neq j$,

$$
\begin{aligned}
w_{i} w_{j}^{*} w_{i} & =p u_{i}\left(p u_{j}\right)^{*} p u_{i}=p u_{i} u_{j}^{*} p u_{i} \\
& =p u_{i} u_{j}^{*}\left(\sum_{|J|=i_{R}}\left(u u^{*}\right)_{J}\right) u_{i}=0,
\end{aligned}
$$

since if $i \in J, u_{i} u_{j}^{*}\left(u u^{*}\right)_{J}=0$ and if $i \notin J$, then $\left(u u^{*}\right)_{J} u_{i}=0$.

Similarly,

$$
w_{i} w_{i}^{*} w_{i}=p u_{i}\left(p u_{i}\right)^{*} p u_{i}=p u_{i}^{*} u_{i} p u_{i}=\left(\sum_{|J|=i_{R}, i \in J}\left(u u^{*}\right)_{J}\right) u_{i}
$$

and $w_{i}=p u_{i}=\sum_{|J|=i_{R}}\left(u u^{*}\right)_{J} u_{i}=\sum_{|J|=i_{R}, i \in J}\left(u u^{*}\right)_{J} u_{i}$, so that $w_{i} w_{i}^{*} w_{i}=w_{i}$.

Now we shall show that $w_{i}$ and $w_{k}$ are colinear. It suffices to show that for $i \neq k$,

$$
p u_{i}\left(p u_{i}\right)^{*} p u_{k}+p u_{k}\left(p u_{i}\right)^{*} p u_{i}=p u_{k},
$$

equivalently (by using Lemma 5.4 on the middle term),

$$
p u_{i} u_{i}^{*} p u_{k} u_{k}^{*}+p u_{k} u_{i}^{*} p u_{i} u_{k}^{*}=p u_{k} u_{k}^{*} .
$$

As noted above,

$$
p u_{k} u_{k}^{*}=p u_{k} u_{k}^{*} p=\sum_{|J|=i_{R}, k \in J}\left(u u^{*}\right)_{J} .
$$

On the other hand we have

$$
p u_{i} u_{i}^{*} p u_{k} u_{k}^{*}=\sum_{|J|=i_{R}, i, k \in J}\left(u u^{*}\right)_{J} .
$$

and

$$
p u_{k} u_{i}^{*} p u_{i} u_{k}^{*}=\left(\sum_{|J|=i_{R}, i \notin J}\left(u u^{*}\right)_{J}\right) u_{k} u_{i}^{*}\left(\sum_{|J|=i_{R}, k \notin J}\left(u u^{*}\right)_{J}\right) u_{i} u_{k}^{*} .
$$

It remains to show that the right side of (21), call it $A$, when added to the right side of (20), equals the right side of (19). 
We have

$$
\begin{aligned}
A & =\left(\sum_{\left|J_{1}\right|=i_{R}, i \notin J_{1}}\left(u u^{*}\right)_{J_{1}}\right) u_{k} u_{i}^{*}\left(\sum_{\left|J_{2}\right|=i_{R}, k \notin J_{2}}\left(u u^{*}\right)_{J_{2}}\right) u_{i} u_{k}^{*} \\
& =\sum_{\left|J_{1}\right|=\left|J_{2}\right|=i_{R}, i \notin J_{1}, k \notin J_{2}}\left(u u^{*}\right)_{J_{1}} u_{k} u_{i}^{*}\left(u u^{*}\right)_{J_{2}} u_{i} u_{k}^{*}
\end{aligned}
$$

Now each term in this sum for which $k \notin J_{1}$ is zero, as is each term for which $i \notin J_{2}$. On the other hand, if $i \in J_{2}$ and $k \in J_{1}$, then by Lemma 5.4,

$$
\left(u u^{*}\right)_{J_{1}} u_{k} u_{i}^{*}\left(u u^{*}\right)_{J_{2}} u_{i} u_{k}^{*}=\left(u u^{*}\right)_{J_{1}}\left(u u^{*}\right)_{J_{2}-\{i\}} u_{k} u_{i}^{*} u_{i} u_{k}^{*}
$$

which is zero unless $J_{1}=J_{2} \cup\{k\}$, in which case it equals $\left(u u^{*}\right)_{J_{1}} u_{k} u_{i}^{*} u_{i} u_{k}$, where $k \in J_{1}$ and $i \notin J_{1}$.

Conversely, if $k \in J$ and $i \notin J$, then

$$
\left(u u^{*}\right)_{J} u_{k} u_{i}^{*} u_{i} u_{k}^{*}=\left(u u^{*}\right)_{J_{1}} u_{k} u_{i}^{*}\left(u u^{*}\right)_{J_{2}} u_{i} u_{k}^{*}
$$

where $J_{1}=J, J_{2}=(J-\{k\}) \cup\{i\},\left|J_{1}\right|=\left|J_{2}\right|=i_{R}, i \notin J_{1}, k \notin J_{2}$. Therefore

$$
\begin{aligned}
A & =\sum_{|J|=i_{R}, k \in J, i \notin J}\left(u u^{*}\right)_{J} u_{k} u_{i}^{*} u_{i} u_{k}^{*} \\
& =\sum_{|J|=i_{R}, k \in J, i \notin J}\left(u u^{*}\right)_{J}\left(u_{k}-u_{i} u_{i}^{*} u_{k}\right) u_{k}^{*} \\
& =\sum_{|J|=i_{R}, k \in J, i \notin J}\left(u u^{*}\right)_{J} u_{k} u_{k}^{*} \\
& =\sum_{|J|=i_{R}, k \in J, i \notin J}\left(u u^{*}\right)_{J},
\end{aligned}
$$

as required. This proves that $\left\{p_{R} u_{k}\right\}_{k=1}^{n}$ is a rectangular rank 1 grid.

Let us now prove that $(1-p) u_{i} \top(1-p) u_{k}$, that is,

$$
(1-p) u_{i}\left[(1-p) u_{i}\right]^{*}(1-p) u_{k}+(1-p) u_{k}\left[(1-p) u_{i}\right]^{*}(1-p) u_{i}=(1-p) u_{k} .
$$

As before, it suffices to prove

$$
(1-p) u_{i} u_{i}^{*}(1-p) u_{k} u_{k}^{*}+(1-p) u_{k} u_{i}^{*}(1-p) u_{i} u_{k}^{*}=(1-p) u_{k} u_{k}^{*}
$$

For the first term on the left side of (22),

$$
\begin{aligned}
& \quad(1-p) u_{i} u_{i}^{*}(1-p) u_{k} u_{k}^{*}= \\
& \quad=(1-p) u_{i} u_{i}^{*} u_{k} u_{k}^{*}-(1-p) u_{i} u_{i}^{*} p u_{k} u_{k}^{*} \\
& \quad=(1-p) u_{i} u_{i}^{*} u_{k} u_{k}^{*}\left(\text { since } u_{i} u_{i}^{*} \text { commutes with } p\right)
\end{aligned}
$$


For the second term on the left side of 22 ,

$$
\begin{aligned}
(1-p) u_{k} u_{i}^{*}(1-p) u_{i} u_{k}^{*}= & (1-p) u_{k} u_{i}^{*} u_{i} u_{k}^{*}-(1-p) u_{k} u_{i}^{*} p u_{i} u_{k}^{*} \\
= & (1-p) u_{k} u_{i}^{*} u_{i} u_{k}^{*}-(1-p) u_{k} u_{i}^{*}\left(\sum_{|J|=i_{R}}\left(u u^{*}\right)_{J}\right) u_{i} u_{k}^{*} \\
= & (1-p) u_{k} u_{i}^{*} u_{i} u_{k}^{*}-(1-p) u_{k} u_{i}^{*}\left(\sum_{|J|=i_{R}, i \in J, k \notin J}\left(u u^{*}\right)_{J}\right) u_{i} u_{k}^{*} \\
= & (1-p) u_{k} u_{i}^{*} u_{i} u_{k}^{*}-(1-p) u_{k} u_{i}^{*}\left(\sum_{|J|=i_{R}-1, i \notin J, k \notin J}\left(u u^{*}\right)_{J}\right) u_{i} u_{k}^{*} \\
= & (1-p) u_{k} u_{i}^{*} u_{i} u_{k}^{*}-(1-p)\left(\sum_{|J|=i_{R}-1, i \notin J, k \notin J}\left(u u^{*}\right)_{J}\right) u_{k} u_{i}^{*} u_{i} u_{k}^{*} \\
= & (1-p) u_{k} u_{i}^{*} u_{i} u_{k}^{*}-(1-p)\left(\sum_{|J|=i_{R}, i \notin J, k \in J}\left(u u^{*}\right)_{J}\right) u_{k} u_{i}^{*} u_{i} u_{k}^{*} \\
= & (1-p) u_{k} u_{i}^{*} u_{i} u_{k}^{*}-(1-p) p u_{k} u_{i}^{*} u_{i} u_{k}^{*} \\
= & (1-p) u_{k} u_{i}^{*} u_{i} u_{k}^{*} .
\end{aligned}
$$

By (23) and (24), the left side of (22) is equal to

$$
(1-p) u_{k} u_{k}^{*} u_{i} u_{i}^{*}+(1-p) u_{k} u_{i}^{*} u_{i} u_{k}^{*}=(1-p) u_{k}\left(u_{k}^{*} u_{i} u_{i}+u_{i}^{*} u_{i} u_{k}^{*}\right)=(1-p) u_{k} u_{k}^{*},
$$

as required.

We omit the analogous proof that $(1-p) u_{i}$ is a minimal partial isometry.

Finally we show that $p Y \perp(1-p) Y$. It suffices to show that basis elements are orthogonal, that is $p u_{i}\left[(1-p) u_{j}\right]^{*}=0$ for all $i, j$. First, if $i \neq j$, then

$$
\begin{aligned}
p u_{i}\left[(1-p) u_{j}\right]^{*} & =p u_{i} u_{j}^{*}(1-p) \\
& =\left(\sum_{i, j \notin J,|J|=i_{R}-1}\left(u u^{*}\right)_{J}\right) u_{i} u_{j}^{*}(1-p) \\
& =u_{i} u_{j}^{*}\left(\sum_{i, j \notin J,|J|=i_{R}-1}\left(u u^{*}\right)_{J}\right)(1-p) \\
& =u_{i} u_{j}^{*}\left(\sum_{i \notin J,|J|=i_{R}}\left(u u^{*}\right)_{J}\right)(1-p) \\
& =u_{i} u_{j}^{*} p(1-p)=0 .
\end{aligned}
$$

Next, $p u_{i}\left[(1-p) u_{i}\right]^{*}=p u_{i} u_{i}^{*}-p u_{i} u_{i}^{*} p=p u_{i} u_{i}^{*}-p u_{i} u_{i}^{*}=0$.

Clearly, $(p z)^{*}(1-p) y=0$ for all $y, z \in Y$.

The next proposition proves Theorem 1 1 (c) (See Remark 6.2 for the definition of the spaces $H_{n}^{k}$ ). 
Proposition 5.10. If either of $i_{R}$ or $i_{L}$ is equal to 1 or $n$, then $Y$ is completely semi-isometric to $R_{n}$ or to $C_{n}$.

Proof. If $i_{R}=1$, then $u_{1}^{*} u_{2}=0$ so by Corollary 5.3(b), $Y$ is completely isometric to $B\left(\mathbb{C}^{n}, \mathbb{C}\right)$. If $i_{L}=1$, then $u_{1} u_{2}^{*}=0$ so by Corollary $5.3(\mathrm{a}), Y$ is completely isometric to $B\left(\mathbb{C}, \mathbb{C}^{n}\right)$.

If $i_{R}=n$, then with $p=p_{R}$,

$$
p u_{1}\left(p u_{2}\right)^{*}=p u_{1} u_{2}^{*} p=\left(u u^{*}\right)_{\{1,2, \ldots, n\}} u_{1} u_{2}^{*} p=0
$$

since $u_{2}^{*} u_{1} u_{2}^{*}=0$, so by Corollary 5.3 (a), $p Y$ is completely isometric to $B\left(\mathbb{C}, \mathbb{C}^{n}\right)$ and by Lemma 5.9, $Y$ is completely semi-isometric to $B\left(\mathbb{C}, \mathbb{C}^{n}\right)$. Similarly, if $i_{L}=n$, then $Y$ is completely semi-isometric to $B\left(\mathbb{C}^{n}, \mathbb{C}\right)$.

In preparation for the next two sections, let us consider the remaining case where $1<i_{R}, i_{L}<n$.

Lemma 5.11. In general, $i_{R}+i_{L} \geq n+1$. Let $p=p_{R}$ and $w_{j}=p u_{j}$. Let $i_{L}^{\prime}$ and $i_{R}^{\prime}$ denote the corresponding indices for the grid $\left\{w_{1}, \ldots, w_{n}\right\}$. Then $i_{L}^{\prime}+i_{R}^{\prime}=n+1$.

Proof. Note first that if $i_{L}<n$,

$$
\begin{aligned}
\left(u^{*} u\right)_{\left\{1,2, \ldots, i_{L}\right\}} & =\left(u^{*} u\right)_{\left\{1,2, \ldots, i_{L}-1\right\}} u_{i_{L}}^{*} u_{i_{L}} \\
& =\left(u^{*} u\right)_{\left\{1,2, \ldots, i_{L}-1\right\}} u_{i_{L}}^{*}\left(u_{i_{L}} u_{i_{L}+1}^{*} u_{i_{L}+1}+u_{i_{L}+1} u_{i_{L}+1}^{*} u_{i_{L}}\right) \\
& =0+\left(u^{*} u\right)_{\left\{1,2, \ldots, i_{L}-1\right\}} u_{i_{L}}^{*} u_{i_{L}+1} u_{i_{L}+1}^{*} u_{i_{L}} \\
& =\left(u^{*} u\right)_{\left\{1,2, \ldots, i_{L}-1\right\}} u_{i_{L}}^{*} u_{i_{L}+1} u_{i_{L}+1}^{*}\left(u_{i_{L}} u_{i_{L}+2}^{*} u_{i_{L}+2}+u_{i_{L}+2} u_{i_{L}+2}^{*} u_{i_{L}}\right) \\
& \left.=\left(u^{*} u\right)_{\left\{1,2, \ldots, i_{L}-1\right\}} u_{i_{L}}^{*}\left(u_{i_{L}+1} u_{i_{L}+1}^{*} u_{i_{L}+2} u_{i_{L}+2}^{*}\right) u_{i_{L}}\right) \\
& =\ldots \\
& =\left(u^{*} u\right)_{\left\{1,2, \ldots, i_{L}-1\right\}} u_{i_{L}}^{*}\left(u u^{*}\right)_{\left\{i_{L}+1, i_{L}+2, \ldots, n\right\}} u_{i_{L}} . \\
& =\left(u^{*} u\right)_{\left\{1,2, \ldots, i_{L}-1\right\}} u_{i_{L}}^{*}\left(u u^{*}\right)_{\left\{i_{L}, i_{L}+1, i_{L}+2, \ldots, n\right\}} u_{i_{L}} .
\end{aligned}
$$

If $n-i_{L}+1>i_{R}$, then $\left|\left\{i_{L}, i_{L}+1, \ldots, n\right\}\right|>i_{R}$, so $\left(u u^{*}\right)_{\left\{1,2, \ldots, i_{L}\right\}}=0$, which is impossible. Hence $i_{L}+i_{R} \geq n+1$, proving the first statement.

It is easy to see that $\left(w^{*} w\right)_{J}=\left(u^{*} u\right)_{J}$ and therefore that $i_{L}^{\prime}=i_{L}$. Indeed, for any $r \geq 1$,

$$
\begin{aligned}
\left(w^{*} w\right)_{\{1,2, \ldots, r\}} & =p u_{1}^{*} u_{1} p u_{2}^{*} u_{2} \cdots p u_{r}^{*} u_{r} p \\
& =\sum_{|J|=i_{L},\{1, \ldots, r\} \subset J}\left(u^{*} u\right)_{J} .
\end{aligned}
$$

Moreover, for any $r \geq 1$,

$$
\begin{aligned}
\left(w w^{*}\right)_{\{1, \ldots, r\}} & =u_{1} p u_{1}^{*} u_{2} p u_{2}^{*} \cdots u_{r} p u_{r}^{*} \\
& =\sum\left[u_{1}\left(u^{*} u\right)_{J_{1}} u_{1}^{*}\right] \cdots\left[u_{r}\left(u^{*} u\right)_{J_{r}} u_{r}^{*}\right]
\end{aligned}
$$

where the sum can be taken over all $\left|J_{k}\right|=i_{L}$ with $k \in J_{k}$ and $(\{1,2, \ldots, r\}-$ $\{k\}) \cap J_{k}=\emptyset$. Indeed, if $k \notin J_{k}$, then $u_{k}\left(u^{*} u\right)_{J_{k}} u_{k}=0$; and if there is a $j \in$ $(\{1,2, \ldots, r\}-\{k\}) \cap J_{k}$, then by commutativity of the factors in the terms of (26), that term would vanish by (18).

Thus if $r \leq i_{R}^{\prime}$, we have $i_{L}=\left|J_{k}\right| \leq n-(r-1)$ and in particular, $i_{L} \leq n-\left(i_{R}^{\prime}-1\right)$, that is, $i_{L}+i_{R}^{\prime} \leq n+1$. Since $i_{L}=i_{L}^{\prime}$ and $i_{R}^{\prime}+i_{L}^{\prime} \geq n+1$, we conclude that $i_{L}^{\prime}+i_{R}^{\prime}=n+1$. 


\section{The Hilbertian operator spaces $H_{n}^{k}$}

In this section we shall begin by assuming that $Y$ is a $J W^{*}$-triple of rank 1 and finite dimension $n$ given by a rectangular rank 1 grid $\left\{u_{1}, \ldots, u_{n}\right\}$ such that $i_{R}+i_{L}=n+1$. If $i_{L}=1$ or if $i_{R}=1$, then $Y$ is completely isometric to the type 1 Cartan factors $R_{n}$ or $C_{n}$ by Corollary 5.3. Otherwise, we shall show in section 7 that $Y$ is completely isometric to a space $H_{n}^{i_{R}}$ which is a subtriple of a Cartan factor of type 1, proving Proposition 2.6 in this case. This will be achieved by constructing, from the given grid $\left\{u_{j}\right\}$, a rectangular grid $\left\{u_{I J}\right\}$ whose linear span is a ternary algebra containing $Y$ and which is ternary isomorphic to a Cartan factor of type 1 , namely the $\left(\begin{array}{c}n \\ i_{L}\end{array}\right)$ by $\left(\begin{array}{c}n \\ i_{R}\end{array}\right)$ complex matrices.

After this, in section 7 we shall prove Proposition 2.6 in case $Y$ is infinite dimensional and of rank 1 .

Here is the construction. We define some elements which are indexed by an arbitrary pair of subsets $I, J$ of $\{1, \ldots, n\}$ satisfying

$$
|I|=i_{R}-1,|J|=i_{L}-1 \text {. }
$$

Note that the number of possible sets $I$ is $\left(\begin{array}{c}n \\ i_{R}-1\end{array}\right)\left(=\left(\begin{array}{c}n \\ i_{L}\end{array}\right)\right)$ and the number of such $J$ is $\left(\begin{array}{c}n \\ i_{L}-1\end{array}\right)\left(=\left(\begin{array}{c}n \\ i_{R}\end{array}\right)\right)$. Moreover, if $|I \cap J|=s \geq 0$, then $\left|(I \cup J)^{c}\right|=s+1$. Hence we may write

$$
I=\left\{i_{1}, \ldots, i_{k}, d_{1}, \ldots, d_{s}\right\} \quad, \quad J=\left\{j_{1}, \ldots, j_{l}, d_{1}, \ldots, d_{s}\right\}
$$

where $I \cap J=\left\{d_{1}, \ldots, d_{s}\right\}$. Let us write $(I \cup J)^{c}=\left\{c_{1}, \ldots, c_{s+1}\right\}$, and let us agree (for the moment) that the elements are ordered as follows: $c_{1}<c_{2}<\cdots<c_{s+1}$ and $d_{1}<d_{2}<\cdots<d_{s}$.

Definition 6.1. With the above notation, we define

$$
u_{I J}=u_{I, J}=\left(u u^{*}\right)_{I-J} u_{c_{1}} u_{d_{1}}^{*} u_{c_{2}} u_{d_{2}}^{*} \cdots u_{c_{s}} u_{d_{s}}^{*} u_{c_{s+1}}\left(u^{*} u\right)_{J-I} .
$$

Remark 6.2. We are going to show (c.f. Proposition 6.9 and 6.10) that there is a choice of signs $\epsilon(I, J)= \pm 1$ such that the family $\left\{\epsilon(I, J) u_{I, J}\right\}$ forms a rectangular grid which is closed under the ternary product $(a, b, c) \mapsto a b^{*} c$, so that its linear span is ternary isomorphic and therefore completely isometric to a concrete Cartan factor of type 1. By restriction, from (34) below, $Y$ will be completely isometric to its image, which we shall denote by $H_{n}^{i_{R}}$. We will then show that all $H_{n}^{k}$ are actually rank 1 triples (and thus Hilbertian) and satisfy $k=i_{R}, i_{R}+i_{L}=n+1$, thus proving the existence of the Hilbert spaces discussed in this section (see the paragraph preceding Example 1 in section 7).

Proposition 6.3. Let $u_{I, J}$ be defined by 28). Then

(a): $u_{I, J}$ is a minimal partial isometry, that is

$$
u_{I, J}\left[u_{I, J}\right]^{*} u_{I, J}=u_{I, J} \text { and } u_{I, J}\left[u_{I^{\prime}, J^{\prime}}\right]^{*} u_{I, J}=0 \text { for all }(I, J) \neq\left(I^{\prime}, J^{\prime}\right) .
$$

(b): orthogonality: $u_{I, J} \perp u_{I^{\prime}, J^{\prime}}$ if $I \neq I^{\prime}$ and $J \neq J^{\prime}$.

(c): colinearity: $u_{I, J} \top_{I^{\prime}, J^{\prime}}$ if either $I=I^{\prime}$ or $J=J^{\prime}$ (but not both).

(d): associative orthogonality:

$$
u_{I, J}\left[u_{I^{\prime}, J^{\prime}}\right]^{*}=0 \text { if } I \neq I^{\prime} \text {; and }\left[u_{I, J}\right]^{*} u_{I^{\prime}, J^{\prime}}=0 \text { if } J \neq J^{\prime} .
$$

(e): “weak" quadrangle property: $u_{I, J}\left[u_{I, J^{\prime}}\right]^{*} u_{I^{\prime}, J^{\prime}}= \pm u_{I^{\prime}, J}$ 
Proof. Throughout this proof, we use the fact that all elements of the grid $\left\{u_{1}, \ldots, u_{n}\right\}$ are present in each $u_{I J}$. To avoid cumbersome notation we will also often denote an element $u_{c}$, where $c \in(I \cup J)^{c}$, by $c_{i j}$, and similarly for $u_{d}$. For example, in (29) below, $d_{i j^{\prime}}^{1}$ denotes $u_{d_{i j^{\prime}}^{1}}^{*}$ where $d_{i j^{\prime}}^{1} \in I \cap J^{\prime}$, and $c_{i j}^{1}$ denotes $u_{c_{i j}^{1}}$, where $c_{i j}^{1} \in(I \cup J)^{c}$.

Proof of (e): By definition,

$$
\begin{aligned}
u_{I, J}\left[u_{I, J^{\prime}}\right]^{*} u_{I^{\prime}, J^{\prime}} & =\left[\left(u u^{*}\right)_{I-J} c_{i j}^{1} d_{i j}^{1} \cdots d_{i j}^{q} c_{i j}^{q+1}\left(u^{*} u\right)_{J-I}\right] \\
& \times\left[\left(u^{*} u\right)_{J^{\prime}-I} c_{i j^{\prime}}^{r+1} d_{i j^{\prime}}^{r} \cdots d_{i j^{\prime}}^{1} c_{i j^{\prime}}^{1}\left(u u^{*}\right)_{I-J^{\prime}}\right] \\
& \times\left[\left(u u^{*}\right)_{I^{\prime}-J^{\prime}} c_{i^{\prime} j^{\prime}}^{1} d_{i^{\prime} j^{\prime}}^{1} \cdots d_{i^{\prime} j^{\prime}}^{s} c_{i^{\prime} j^{\prime}}^{s+1}\left(u^{*} u\right)_{J^{\prime}-I^{\prime}}\right] .
\end{aligned}
$$

This quantity remains unchanged if the factors

$$
\left(u^{*} u\right)_{J-I}\left(u^{*} u\right)_{J^{\prime}-I} \text { and }\left(u u^{*}\right)_{I-J^{\prime}}\left(u u^{*}\right)_{I^{\prime}-J^{\prime}}
$$

are removed. Indeed, since $J-I \subset\left(I^{c} \cap{J^{\prime}}^{c}\right) \cup\left(I^{c} \cap J^{\prime}\right.$ (disjoint union), by using Lemma $5.4\left(u^{*} u\right)_{J-I}$ can be absorbed into the $c_{i j^{\prime}}$ 's or into $\left(u u^{*}\right)_{J^{\prime}-I}$. Similarly, $\left(u u^{*}\right)_{I^{\prime}-J^{\prime}}$ can be absorbed into the $c_{i j^{\prime}}$ 's or into $\left(u u^{*}\right)_{I-J^{\prime}}$. After this has been done, $\left(u^{*} u\right)_{J^{\prime}-I}$ can be absorbed into the $d_{i^{\prime} j^{\prime}}$ 's or into $\left(u^{*} u\right)_{J^{\prime}-I^{\prime}}$, and $\left(u u^{*}\right)_{I-J^{\prime}}$ can be absorbed into the $d_{i j}$ 's or into $\left(u u^{*}\right)_{I-J}$,

Thus

$u_{I, J}\left[u_{I, J^{\prime}}\right]^{*} u_{I^{\prime}, J^{\prime}}=$

$$
\left(u u^{*}\right)_{I-J} c_{i j}^{1} d_{i j}^{1} \cdots d_{i j}^{q} c_{i j}^{q+1} c_{i j^{\prime}}^{r+1} d_{i j^{\prime}}^{r} \cdots d_{i j^{\prime}}^{1} c_{i j^{\prime}}^{1} c_{i^{\prime} j^{\prime}}^{1} d_{i^{\prime} j^{\prime}}^{1} \cdots d_{i^{\prime} j^{\prime}}^{s} c_{i^{\prime} j^{\prime}}^{s+1}\left(u^{*} u\right)_{J^{\prime}-I^{\prime}} .
$$

We claim next that in fact

$$
u_{I, J}\left[u_{I, J^{\prime}}\right]^{*} u_{I^{\prime}, J^{\prime}}=
$$

$$
\left(u u^{*}\right)_{I^{\prime}-J} c_{i j}^{1} d_{i j}^{1} \cdots d_{i j}^{q} c_{i j}^{q+1} c_{i j^{\prime}}^{r+1} d_{i j^{\prime}}^{r} \cdots d_{i j^{\prime}}^{1} c_{i j^{\prime}}^{1} c_{i^{\prime} j^{\prime}}^{1} d_{i^{\prime} j^{\prime}}^{1} \cdots d_{i^{\prime} j^{\prime}}^{s} c_{i^{\prime} j^{\prime}}^{s+1}\left(u^{*} u\right)_{J-I^{\prime}} .
$$

To get from (30) to (31) we proceed as follows. Consider first an element $x \in$ $I^{\prime}-J$. Either $x \in I$ or $x \notin I$. In the latter case, $x \in(I \cup J)^{c}$ so that $u_{x}$ is one of the $c_{i j}$ and so $u_{x} u_{x}^{*}$ can be split off from $u_{x}=u_{x} u_{x}^{*} u_{x}$ and absorbed (using Lemma 5.4) into the $\left(u u^{*}\right)_{I-J}$ term. In the former case, no absorption is necessary. Doing this for every such $x$ allows us to replace the term $\left(u u^{*}\right)_{I-J}$ in 330 by $\left(u u^{*}\right)_{\left(I \cup I^{\prime}\right)-J}$.

We now have

$u_{I, J}\left[u_{I, J^{\prime}}\right]^{*} u_{I^{\prime}, J^{\prime}}=$

(32) $\left(u u^{*}\right)_{\left(I \cup I^{\prime}\right)-J} c_{i j}^{1} d_{i j}^{1} \cdots d_{i j}^{q} c_{i j}^{q+1} c_{i j^{\prime}}^{r+1} d_{i j^{\prime}}^{r} \cdots d_{i j^{\prime}}^{1} c_{i j^{\prime}}^{1} c_{i^{\prime} j^{\prime}}^{1} d_{i^{\prime} j^{\prime}}^{1} \cdots d_{i^{\prime} j^{\prime}}^{s} c_{i^{\prime} j^{\prime}}^{s+1}\left(u^{*} u\right)_{J^{\prime}-I^{\prime}}$.

Now consider an element $x \in I-J$. Either $x \in J^{\prime}$ or $x \notin J^{\prime}$. In the first case, $x \in I \cap J^{\prime}$ so that $u_{x}$ is one of the $d_{i j^{\prime}}$ and therefore any such $u_{x} u_{x}^{*}$ can be absorbed from the term $\left(u u^{*}\right)_{\left(I \cup I^{\prime}\right)-J}$ into a $d_{i j^{\prime}}$. On the other hand, if $x \notin J^{\prime}$, then either $x \in I^{\prime}$, in which case no absorption is necessary, or $x \notin I^{\prime}$ so that $x \in\left(I^{\prime} \cup J^{\prime}\right)^{c}$ and $u_{x}$ is one of the $c_{i^{\prime} j^{\prime}}$ and hence $u_{x} u_{x}^{*}$ can be absorbed. Doing this for every such $x$ allows us to replace the term $\left(u u^{*}\right)_{\left(I \cup I^{\prime}\right)-J}$ in (32) by $\left(u u^{*}\right)_{I^{\prime}-J}$.

By an entirely similar two-step argument, we may replace $\left(u^{*} u\right)_{J^{\prime}-I^{\prime}}$ in (30) by $\left(u^{*} u\right)_{J-I^{\prime}}$, which proves (31). 
To complete the proof of (e), we need to show that the right side of (31) has the form

$$
\pm\left(u u^{*}\right)_{I^{\prime}-J} c_{i^{\prime} j}^{1} d_{i^{\prime} j}^{1} \cdots d_{i^{\prime} j}^{t} c_{i^{\prime} j}^{t+1}\left(u^{*} u\right)_{J-I^{\prime}}
$$

To do this we must examine each of the elements $c_{i j}, d_{i j^{\prime}}, c_{i^{\prime} j^{\prime}}$ in (31) (call them "outer" elements as they are not "starred") and $d_{i j}, c_{i j^{\prime}}, d_{i^{\prime} j^{\prime}}$ (call them "inner" elements, as they are "starred") and decide whether to leave the element there or absorb it into one of the end terms $\left(u^{*} u\right)_{J-I^{\prime}}$ or $\left(u u^{*}\right)_{I^{\prime}-J}$. This is achieved in the following lemma.

Lemma 6.4. Retain the above notation.

(a): Each "outer" element $c_{i j}, d_{i j^{\prime}}, c_{i^{\prime} j^{\prime}}$ on the right side of (31) is either equal to $a c_{i^{\prime} j}$ or is equal to a unique other element on the right side of (31), together with which it can be absorbed into one of the terms $\left(u^{*} u\right)_{J-I^{\prime}}$ or $\left(u u^{*}\right)_{I^{\prime}-J}$. Conversely, every $c_{i^{\prime} j}$ is equal to one of these "outer" elements.

(b): Similarly, each "inner" element $d_{i j}, c_{i j^{\prime}}, d_{i^{\prime} j^{\prime}}$ is either equal to a $d_{i^{\prime} j}$ or is equal to a unique other element, together with which it can be absorbed into one of the terms $\left(u^{*} u\right)_{J-I^{\prime}}$ or $\left(u u^{*}\right)_{I^{\prime}-J}$. Conversely, every $d_{i^{\prime} j}$ is equal to one of these "inner" elements.

Proof of Lemma 6.4. For three mutually colinear partial isometries $u, v, w$, the term "flipping" in this proof refers to the fact that $u v^{*} w=-w v^{*} u$.

Let $c_{i j} \in(I \cup J)^{c}$. Either $c_{i j} \in I^{\prime}$ or $c_{i j} \notin I^{\prime}$. In the first case $c_{i j}$ is a $c_{i^{\prime} j}$ and no absorption is necessary. In the second case, either $c_{i j} \in J^{\prime}$ or $c_{i j} \notin J^{\prime}$. If the former, $c_{i j} \in I^{\prime} \cap J^{\prime}$ so that $c_{i j}$ is equal to a $d_{i^{\prime} j^{\prime}}$ with which it can be paired by "flipping" and $c_{i j} c_{i j}^{*}$ can be absorbed into $\left(u u^{*}\right)_{I^{\prime}-J}$ by Lemma 5.4. In the latter, $c_{i j} \in\left(I \cup J^{\prime}\right)^{c}$ so that $c_{i j}$ is equal to a $c_{i j}$, with which it can be paired and absorbed as above by repeated use of Lemma 5.4 .

Let $d_{i j^{\prime}} \in I \cap J^{\prime}$. Either $d_{i j^{\prime}} \notin I^{\prime}$ or $d_{i j^{\prime}} \in I^{\prime}$. In the second case $d_{i j^{\prime}} \in I^{\prime}$ so that $d_{i j^{\prime}} \in I^{\prime} \cap J^{\prime}$ and $d_{i j^{\prime}}$ is equal to a $d_{i^{\prime} j^{\prime}}$ so can be flipped and absorbed. In the first case, either $d_{i j^{\prime}} \notin J$, in which case it is a $c_{i j^{\prime}}$ and no absorption is necessary, or $d_{i j^{\prime}} \in J$ so that $d_{i j^{\prime}}$ is equal to a $d_{i j}$ and can be flipped and absorbed into $\left(u^{*} u\right)_{J-I^{\prime}}$.

The proof for the third type of "inner" element, as well as the proofs for the "outer" elements are similar.

For the converse statement in (a), note that $c_{i^{\prime} j} \in I^{\prime c} \cap J^{c} \subset\left(I^{c} \cap J^{c}\right) \cup(I \cap$ $\left.J^{\prime}\right) \cup\left(I^{\prime c} \cap J^{\prime c}\right)$ and $d_{i^{\prime} j} \in I^{\prime} \cap J \subset(I \cap J) \cup\left(I^{c} \cap J^{\prime c}\right) \cup\left(I^{\prime} \cap J^{\prime}\right)$.

With Lemma 6.4, the proof of (e) is completed.

Proof of (d): If we let $w$ denote $u_{I, J}\left[u_{I^{\prime}, J^{\prime}}\right]^{*}$, then

$w=\left[\left(u u^{*}\right)_{I-J} c_{i j}^{1} d_{i j}^{1} \cdots d_{i j}^{s} c_{i j}^{s+1}\left(u^{*} u\right)_{J-I}\right]\left[\left(u^{*} u\right)_{J^{\prime}-I^{\prime}} c_{i^{\prime} j^{\prime}}^{r+1} d_{i^{\prime} j^{\prime}}^{r} \cdots d_{i^{\prime} j^{\prime}}^{1} c_{i^{\prime} j^{\prime}}^{1}\left(u^{*} u\right)_{I^{\prime}-J^{\prime}}\right]$.

Since $I \neq I^{\prime}$, there are two possibilities: either there exists $i_{0} \in I-I^{\prime}$ or there exists $i_{0}^{\prime} \in I^{\prime}-I$. We shall deal with the first case only as the other is similar.

So assume first that $i_{0} \in I-I^{\prime}$ and consider the two cases: $i_{0} \in J$ and $i_{0} \notin J$. In the first case $u_{i_{0}}$ is one of the $d_{i j}$ and hence either $u_{i_{0}}$ is also a $c_{i^{\prime} j^{\prime}}$ in which case $w=0$ by "flipping" and minimality; or $i_{0} \in J^{\prime}-I^{\prime}$ in which case $w=0$ by "hopping" and minimality. 
Now consider the case that $i_{0} \notin J$. In this case $i_{0} \in I-J$ and hence either $i_{0} \in J^{\prime}$, in which case $i_{0} \in J^{\prime}-I^{\prime}$ and $w=0$ by "hopping" and minimality; or $i_{0} \notin J^{\prime}$, in which case $u_{i_{0}}$ is a $c_{i^{\prime} j^{\prime}}$ and $w=0$ again by "hopping" and minimality.

This proves the first statement in (d). The proof of the second statement is achieved in a similar way.

The reader will note that "maximality" (meaning for instance that $\left(u^{*} u\right)_{J}=0$ if $|J|>i_{L}$ ) was not used in the above proof of (d). It's main use is in the proof of the important decomposition (34) below. (c).

It being clear that (a) and (b) follow immediately from (d), it remains to prove

Proof of (c): In view of the strong orthogonality already proved, it will suffice to prove that $u_{I, J}\left[u_{I, J}\right]^{*} u_{I^{\prime}, J}=u_{I^{\prime}, J}$ for $I \neq I^{\prime}$. We have

$$
\begin{aligned}
u_{I, J}\left[u_{I, J}\right]^{*} u_{I^{\prime}, J} & =\left[\left(u u^{*}\right)_{I-J} c_{i j}^{1} d_{i j}^{1} \cdots d_{i j}^{s} c_{i j}^{s+1}\left(u^{*} u\right)_{J-I}\right] \\
& \times\left[c_{i j}^{s+1} d_{i j}^{s} \cdots d_{i j}^{1} c_{i j}^{1}\left(u u^{*}\right)_{I-J}\right] \\
& \times\left[\left(u u^{*}\right)_{I^{\prime}-J} c_{i^{\prime} j}^{1} d_{i^{\prime} j}^{1} \cdots d_{i^{\prime} j}^{r} c_{i^{\prime} j}^{r+1}\left(u^{*} u\right)_{J-I^{\prime}}\right] .
\end{aligned}
$$

The term $\left(u^{*} u\right)_{J-I}$ in (33) can be absorbed into the $d_{i^{\prime} j}$ 's or into $\left(u^{*} u\right)_{J-I^{\prime}}$ by Lemma 5.4. Then in turn, the products $c_{i j}^{s+1}\left(c_{i j}^{s+1}\right)^{*}, d_{i j}^{s}\left(d_{i j}^{s}\right)^{*}, \ldots, c_{i j}^{1}\left(c_{i j}^{1}\right)^{*}$ can be alternatingly absorbed into the combination of $\left(u u^{*}\right)_{I^{\prime}-J}$ and the $c_{i^{\prime} j}$ 's, or the combination of $\left(u^{*} u\right)_{J-I^{\prime}}$ and the $d_{i^{\prime} j}$ 's.

Finally both of the occurences of the term $\left(u u^{*}\right)_{I-J}$ can also be absorbed into either $\left(u u^{*}\right)_{I^{\prime}-J}$ or a $c_{i^{\prime} j}$, and what remains is $u_{I^{\prime}, J}$. This completes the proof of Proposition 6.3.

Definition 6.5. In the special case of (28) where $I \cap J=\emptyset$, we have $s=0$ and $u_{I, J}$ has the form

$$
u_{I, J}=\left(u u^{*}\right)_{I} u_{c}\left(u^{*} u\right)_{J},
$$

where since $i_{R}+i_{L}=n+1, I \cup J \cup\{c\}=\{1, \ldots, n\}$. We call such an element $a$ "one", and denote it by $u_{I, c, J}$.

Lemma 6.6. For any $c \in\{1, \ldots, n\}$,

$$
u_{c}=\sum_{I, J} u_{I, J}=\sum_{I, J} u_{I, c, J}
$$

where the sum is taken over all disjoint $I, J$ satisfying (27) and not containing $c$.

Proof. For convenience, let us say that for colinear partial isometries $u$ and $v$, the formula $u=u v^{*} v+v v^{*} u$ is the result of "applying $v$ to $u$ ". Given $c$, write $\{1, \ldots, n\}=\left\{c, c_{2}, \ldots, c_{n}\right\}$. The equation (34) is obtained by first applying $u_{c_{2}}$ to $u_{c}$, then applying $u_{c_{3}}$ to all occurrences of $u_{c}$, and in turn applying $u_{c_{4}}, \ldots, u_{c_{n}}$ to all occurences of $u_{c}$ that are created in the previous step.

We thereby obtain

$$
u_{c}=\sum\left(u u^{*}\right)_{I} u_{c}\left(u^{*} u\right)_{J}
$$

where the sum is over all disjoint subsets $I, J$ of $\{1, \ldots, n\}-\{c\}$ with

$$
I \cup J \cup\{c\}=\{1, \ldots, n\} .
$$

A term in this sum is zero unless $|I| \leq i_{R}-1$ and $|J| \leq i_{L}-1$. By (35) and the fact that $i_{R}+i_{L}=n+1,|I|=i_{R}-1$ and $|J|=i_{L}-1$ so (34) follows. 
Note that a change in the order of the $u_{c}$ 's or $u_{d}$ 's in (28) can at most change the sign, since any such change can be accomplished by "flipping." In the next lemma, we consider elements defined by the right side of (28) but without specifying an ordering of the $c$ 's and $d$ 's. This lemma will enable us to define the signature $\epsilon(I, J)$ of $u_{I, J}$ and prove the important Proposition 6.10.

Lemma 6.7. Given $I, J$ with $|I|=i_{R}-1,|J|=i_{L}-1$, let $C=(I \cup J)^{c}$ and $D=I \cap J$. For any permutations $\left(c_{1}, \ldots, c_{s+1}\right)$ of $C$ and $\left(d_{1}, \ldots, d_{s}\right)$ of $D$, the element

$$
\left(u u^{*}\right)_{I-J} u_{c_{1}} u_{d_{1}}^{*} u_{c_{2}} u_{d_{2}}^{*} \cdots u_{c_{s}} u_{d_{s}}^{*} u_{c_{s+1}}\left(u^{*} u\right)_{J-I}
$$

(which equals $\pm u_{I J}$ ) decomposes uniquely as a product of "ones":

$$
\left[u_{I_{1}, c_{1}, J_{1}}\right]\left[u_{K_{1}, d_{1}, L_{1}}\right]^{*}\left[u_{I_{2}, c_{2}, J_{2}}\right]\left[u_{K_{2}, d_{2}, L_{2}}\right]^{*} \cdots\left[u_{I_{s}, c_{s}, J_{s}}\right]\left[u_{K_{s}, d_{s}, L_{s}}\right]^{*}\left[u_{I_{s+1}, c_{s+1}, J_{s+1}}\right],
$$

where the $I_{i}, J_{j}, K_{k}, L_{l}$ are uniquely determined by $I, J$ and the c's and d's.

Proof. Let us first prove the existence. Each of the steps in the following equation array is achieved by "expanding" (for example, $u_{c_{2}}=u_{c_{2}} u_{c_{2}}^{*} u_{c_{2}}$ ) and/or "hopping":

$$
\begin{aligned}
& \left(u u^{*}\right)_{I-J} u_{c_{1}} u_{d_{1}}^{*} u_{c_{2}} u_{d_{2}}^{*} \cdots u_{c_{s}} u_{d_{s}}^{*} u_{c_{s+1}}\left(u^{*} u\right)_{J-I} \\
& =\left(u u^{*}\right)_{(I-J) \cup\left(C-c_{1}\right)} u_{c_{1}}\left[u_{d_{1}}^{*} u_{c_{2}} u_{d_{2}}^{*} \cdots u_{c_{s}} u_{d_{s}}^{*} u_{c_{s+1}}\right]\left(u^{*} u\right)_{(J-I) \cup\left(C-c_{s+1}\right)} \\
& =\left(u u^{*}\right)_{(I-J) \cup\left(C-c_{1}\right)} u_{c_{1}}\left(u^{*} u\right)_{J-I}\left[u_{d_{1}}^{*} u_{c_{2}} u_{d_{2}}^{*} \cdots u_{c_{s}} u_{d_{s}}^{*} u_{c_{s+1}}\right]\left(u^{*} u\right)_{(J-I) \cup\left(C-c_{s+1}\right)} \\
& =\left(u u^{*}\right)_{(I-J) \cup\left(C-c_{1}\right)} u_{c_{1}}\left(u^{*} u\right)_{(J-I) \cup D}\left[u_{d_{1}}^{*} u_{c_{2}} u_{d_{2}}^{*} \cdots u_{c_{s}} u_{d_{s}}^{*} u_{c_{s+1}}\right]\left(u^{*} u\right)_{(J-I) \cup\left(C-c_{s+1}\right)} \\
& =\left(u u^{*}\right)_{(I-J) \cup\left(C-c_{1}\right)} u_{c_{1}}\left(u^{*} u\right)_{(J-I) \cup D} \\
& \quad \times\left[u_{d_{1}}^{*} u_{c_{2}} u_{d_{2}}^{*} \cdots u_{c_{s}} u_{d_{s}}^{*}\left(u u^{*}\right)_{(I-J) \cup D} u_{c_{s+1}}\right]\left(u^{*} u\right)_{(J-I) \cup\left(C-c_{s+1}\right)} \\
& =\left[\left(u u^{*}\right)_{(I-J) \cup\left(C-c_{1}\right)} u_{c_{1}}\left(u^{*} u\right)_{(J-I) \cup D}\right] \\
& \left.\times\left[\left(u^{*} u\right)_{(J-I) \cup\left\{c_{1}\right.}\right\} u_{d_{1}}^{*} u_{c_{2}} u_{d_{2}}^{*} \cdots u_{c_{s}} u_{d_{s}}^{*}\left(u u^{*}\right)_{(I-J) \cup\left\{c_{s+1}\right\}}\right] \\
& \times\left[\left(u u^{*}\right)_{(I-J) \cup D} u_{c_{s+1}}\left(u^{*} u\right)_{(J-I) \cup\left(C-c_{s+1}\right)}\right]
\end{aligned}
$$

This shows that $\left(u u^{*}\right)_{I-J} u_{c_{1}} u_{d_{1}}^{*} u_{c_{2}} u_{d_{2}}^{*} \cdots u_{c_{s}} u_{d_{s}}^{*} u_{c_{s+1}}\left(u^{*} u\right)_{J-I}$ equals

$\left[u_{(I-J) \cup\left(C-c_{1}\right), c_{1},(J-I) \cup D}\right]\left[u_{(I-J) \cup\left\{c_{s+1}\right\},(J-I) \cup\left\{c_{1}\right\}}\right]^{*}\left[u_{(I-J) \cup D, c_{s+1},(J-I) \cup\left(C-c_{s+1}\right)}\right]$, which is of the form $u_{I_{1}, c_{1}, J_{1}}\left[u_{I_{2}, J_{2}}\right]^{*} u_{I_{3}, c_{s+1}, J_{3}}$, so the existence follows by induction.

We now prove the uniqueness. Look at the first three factors of (36). Since $u_{I J} \neq 0$, by Proposition $6.3(\mathrm{~d})$, we must have $I_{1}=K_{1}$ and $L_{1}=J_{2}$. Furthermore, since $I_{1} \cup\left\{c_{1}\right\} \cup J_{1}=K_{1} \cup\left\{d_{1}\right\} \cup L_{1}$, we have $J_{2}=\left(J_{1} \cup\left\{c_{1}\right\}\right)-\left\{d_{1}\right\}$. Continuing, we see that all the sets $I_{i}, J_{j}, K_{k}, L_{l}$ are uniquely determined by $J_{1}$ and the $c$ 's and d's. A close look at (36) and using Proposition 6.3 (d) and (e) reveals that

$$
u_{I J}= \pm u_{I_{1} J_{1}}\left[u_{I_{1} J_{s+1}}\right]^{*} u_{I_{s+1} J_{s+1}}
$$

which equals $\pm u_{I_{s+1} J_{1}}$ by Proposition 6.3 (e), so $u_{I J}\left[u_{I_{s+1} J_{1}}\right]^{*} \neq 0$. Then by Proposition 6.3 (d) again, $I=I_{s+1}$ and similarly $J=J_{1}$, completing the proof of uniqueness.

Definition 6.8. We assign a signature to each "one" as follows: Let the elements of $I$ be $i_{1}<i_{2}<\cdots<i_{p}$ (where $p=i_{R}-1$ ) and the elements of $J$ be $j_{1}<j_{2}<$ $\cdots<j_{q}$ (where $\left.q=i_{L}-1\right)$. Then $\epsilon(I, k, J)$ is defined to be the signature of the permutation taking the $n$-tuple $\left(i_{1}, \ldots, i_{p}, k, j_{1}, \ldots, j_{q}\right)$ onto $(1,2, \ldots, n)$. 
The signature $\epsilon(I, J)$ of $u_{I, J}$ is defined to be the product of the signatures of the factors in its decomposition (36) (Recall that $u_{I J}$ is defined so that the $c$ 's and d's are in increasing order).

The next lemma will consider a 3 -tuple $\left(u_{I, J^{\prime}}, u_{I, J}, u_{I^{\prime}, J}\right)$, with $I \neq I^{\prime}$ and $J \neq J^{\prime}$, so that by Proposition 6.3, $u_{I, J^{\prime}} \perp u_{I^{\prime}, J}, u_{I, J^{\prime}} \top u_{I, J}$ and $u_{I, J} \top u_{I^{\prime}, J}$.

Let us further assume that each element of this 3-tuple is a "one". Then it is clear that $I^{\prime}=(I-\{a\}) \cup\{b\}$ and $J^{\prime}=(J-\{c\}) \cup\{b\}$ for suitable elements $a \in I, c \in J$ and $b \in(I \cup J)^{c}$. Hence the 3 -tuple has the form

$$
\left(u_{I, c,(J-c) \cup\{b\}}, u_{I, b, J}, u_{(I-a) \cup\{b\}, a, J}\right) .
$$

By direct calculation and simplification

$$
u_{I J^{\prime}}\left[u_{I J}\right]^{*} u_{I^{\prime} J}=\left(u u^{*}\right)_{I} u_{c}\left(u^{*} u\right)_{J} u_{b}^{*}\left(u u^{*}\right)_{I} u_{a}\left(u^{*} u\right)_{J} .
$$

Since $a \notin J, b \notin J$ and $I \cap J=\emptyset$, Lemma 5.4 shows that

$$
u_{I J^{\prime}}\left[u_{I J}\right]^{*} u_{I^{\prime} J}=\left(u u^{*}\right)_{I} u_{c} u_{b}^{*}\left(u u^{*}\right)_{I} u_{a}\left(u^{*} u\right)_{J} .
$$

Similarly, since $b \notin I, c \notin I$, we can remove the term $\left(u u^{*}\right)_{I}$ to obtain

$$
u_{I J^{\prime}}\left[u_{I J}\right]^{*} u_{I^{\prime} J}=\left(u u^{*}\right)_{I} u_{c} u_{b}^{*} u_{a}\left(u^{*} u\right)_{J}
$$

which equals $\pm u_{I^{\prime} J^{\prime}}$.

Thus, from (38) and the uniqueness in Lemma 6.7, every such 3-tuple (37) of "ones" uniquely determines a corresponding 3 -tuple of "ones" $\left(u_{I^{\prime \prime} J^{\prime}}, u_{I^{\prime \prime}} J^{\prime \prime}, u_{I^{\prime}} J^{\prime \prime}\right)$, such that

$$
u_{I^{\prime \prime} J^{\prime}}\left[u_{I^{\prime \prime} J^{\prime \prime}}\right]^{*} u_{I^{\prime} J^{\prime \prime}}= \pm u_{I^{\prime} J^{\prime}}=\left(u u^{*}\right)_{I} u_{a} u_{b}^{*} u_{c}\left(u^{*} u\right)_{J},
$$

where $I^{\prime \prime}=(I \cup\{c\})-\{a\}$ and $J^{\prime \prime}=(J \cup\{a\})-\{c\}$. The given 3-tuple and the derived one thus have the forms

$$
\left(u_{I, c,(J-c) \cup\{b\}}, u_{I, b, J}, u_{(I-a) \cup\{b\}, a, J}\right)
$$

and

$$
\left(u_{(I \cup\{c\})-a, a,(J-c) \cup\{b\}}, u_{(I \cup\{c\})-a, b,(J-c) \cup\{a\}}, u_{(I-a) \cup\{b\}, c,(J-c) \cup\{a\}}\right) .
$$

Lemma 6.9. Retain the above notation.

(a): $u_{I J^{\prime}}\left[u_{I J}\right]^{*} u_{I^{\prime} J}=-u_{I^{\prime \prime} J^{\prime}}\left[u_{I^{\prime \prime} J^{\prime \prime}}\right]^{*} u_{I^{\prime} J^{\prime \prime}}$.

(b): $\epsilon\left(I J^{\prime}\right) \epsilon(I J) \epsilon\left(I^{\prime} J\right)=-\epsilon\left(I^{\prime \prime} J^{\prime}\right) \epsilon\left(I^{\prime \prime} J^{\prime \prime}\right) \epsilon\left(I^{\prime} J^{\prime \prime}\right)$.

(c): For every 3-tuple (37) of ones,

$\left[\epsilon\left(I J^{\prime}\right) u_{I J^{\prime}}\right]\left[\epsilon(I J) u_{I J}\right]^{*}\left[\epsilon\left(I^{\prime} J\right) u_{I^{\prime} J}\right]=\left[\epsilon\left(I^{\prime \prime} J^{\prime}\right) u_{I^{\prime \prime} J^{\prime}}\right]\left[\epsilon\left(I^{\prime \prime} J^{\prime \prime}\right) u_{I^{\prime \prime} J^{\prime \prime}}\right]^{*}\left[\epsilon\left(I^{\prime} J^{\prime \prime}\right) u_{I^{\prime} J^{\prime \prime}}\right]$.

Proof.

(a) follows from (38) and (39).

(b) We shall use the more precise notation of (40) and (41). Write

$$
u_{I, b, J}=\left(u u^{*}\right)_{\left\{i_{1}, i_{2}, \ldots, i_{u}, i_{u+1}, \ldots, i_{t}, \ldots, i_{i_{R}-1}\right\}} u_{b}^{*}\left(u^{*} u\right)_{\left\{j_{1}, j_{2}, \ldots, j_{s}, \ldots, j_{v}, j_{v+1}, \ldots, j_{i_{L}-1}\right\}},
$$

where $i_{u}<c<i_{u+1}, i_{t}=a, j_{s}=c$ and $j_{v}<a<j_{v+1}$.

We can calculate $\epsilon(I, b, J) \epsilon\left(I^{\prime \prime}, b, J^{\prime \prime}\right)$ by counting the number of transpositions required in "moving" $a$ from $I$ to $J^{\prime \prime}$ and $c$ from $J$ to $I^{\prime \prime}$. These are

$$
\left(i_{R}-1\right)-t+1+(v+1)
$$

and

$$
(s-1)+1+\left(i_{R}-1\right)-u
$$


respectively.

We can calculate $\epsilon\left(I^{\prime}, a, J\right) \epsilon\left(I^{\prime}, c, J^{\prime \prime}\right)$ by counting the number of transpositions required in "moving" $a$ from the "middle" to $J$ " and $c$ from $J$ to the "middle". Taken together this is

$$
v+(s-1) .
$$

We can calculate $\epsilon\left(I, c, J^{\prime}\right) \epsilon\left(I^{\prime \prime}, a, J^{\prime \prime}\right)$ by counting the number of transpositions required in "moving" $c$ from the "middle" to $I^{\prime \prime}$ and $a$ from $I$ to the "middle". Taken together this is

$$
\left(i_{R}-1\right)-u+\left(i_{R}-1-t\right)
$$

The sum of (42)-(45) is $2(s+v-u-t)-1$ which is odd, so exactly one or three of the numbers

$$
\epsilon(I, b, J) \epsilon\left(I^{\prime \prime}, b, J^{\prime \prime}\right), \epsilon\left(I^{\prime}, a, J\right) \epsilon\left(I^{\prime}, c, J^{\prime \prime}\right), \epsilon\left(I, c, J^{\prime}\right) \epsilon\left(I^{\prime \prime}, a, J^{\prime \prime}\right)
$$

equals -1 . In either case, (b) follows.

(c) follows from (a) and (b).

Proposition 6.10. The family $\left\{\epsilon(I J) u_{I, J}\right\}$ forms a rectangular grid which satisfies

$$
\epsilon(I J) u_{I J}\left[\epsilon\left(I J^{\prime}\right) u_{I J^{\prime}}\right]^{*} \epsilon\left(I^{\prime} J^{\prime}\right) u_{I^{\prime} J^{\prime}}=\epsilon\left(I^{\prime} J\right) u_{I^{\prime} J} .
$$

Proof. Since $u_{I^{\prime} J^{\prime}}\left[u_{I J^{\prime}}\right]^{*}=0$ for $I \neq I^{\prime}$, the property (12) will follow from (46). The other grid properties are contained in Proposition 6.3.

To prove (46), we apply Lemma 6.7 to decompose its left and right sides into "ones." To avoid cumbersome notation let us denote any "one" with $u_{c}$ in the "middle" simply by $(c)$ and its signature by $\epsilon$, with an identifying subscript. With this convention, we have, for suitable $x_{j}, y_{k}, z_{l}, w_{i} \in\{1, \ldots, n\}$ and $\epsilon_{p q}= \pm 1$,

$$
\begin{aligned}
\epsilon(I J) u_{I J}\left[\epsilon\left(I J^{\prime}\right) u_{I J^{\prime}}\right]^{*} \epsilon\left(I^{\prime} J^{\prime}\right) u_{I^{\prime} J^{\prime}} & =\left(\epsilon_{11} x_{1}\right)\left(\epsilon_{12} x_{2}\right) \cdots\left(\epsilon_{1,2 r+1} x_{2 r+1}\right) \\
& \times\left[\left(\epsilon_{21} y_{1}\right)\left(\epsilon_{22} y_{2}\right) \cdots\left(\epsilon_{2,2 s+1} x_{2 s+1}\right)\right]^{*} \\
& \times\left(\epsilon_{31} z_{1}\right)\left(\epsilon_{32} z_{2}\right) \cdots\left(\epsilon_{3,2 t+1} z_{2 t+1}\right),
\end{aligned}
$$

and

$$
\epsilon\left(I^{\prime} J\right) u_{I^{\prime} J}=\left(\epsilon_{41} w_{1}\right)\left(\epsilon_{42} w_{2}\right) \cdots\left(\epsilon_{4,2 n+1} w_{2 n+1}\right) .
$$

Recall that from Proposition 6.3(e), we have

$$
\epsilon(I J) u_{I J}\left[\epsilon\left(I J^{\prime}\right) u_{I J^{\prime}}\right]^{*} \epsilon\left(I^{\prime} J^{\prime}\right) u_{I^{\prime} J^{\prime}}= \pm u_{I^{\prime} J} .
$$

Now each $w_{i}$ is either an $x_{j}, y_{k}$ or $z_{l}$ by the proof of Lemma 6.4, and by that same Lemma, the $x_{j}, y_{k}, z_{l}$ which are not used, call them $v_{1}, \ldots, v_{m}$, occur twice with an even number of elements between them. Also, by Lemma 6.9.(c) we may rearrange terms so that the right side of (47) becomes

$$
\left[\left(\epsilon_{51} v_{1}\right)\left(\epsilon_{51} v_{1}\right) \cdots\left(\epsilon_{5 m} v_{m}\right)\left(\epsilon_{5 m} v_{m}\right)\right]\left[\left(\epsilon_{61} w_{1}\right)^{\prime}\left(\epsilon_{62} w_{2}\right)^{\prime} \cdots\left(\epsilon_{6,2 n+1} w_{2 n+1}\right)^{\prime}\right]
$$

where the notation $(\epsilon w)^{\prime}$ indicates that the $I$ and $J$ in $(w)$ may have changed.

Since $\left(v_{j}\right)\left(v_{j}\right)^{*}\left(w_{1}\right)=\left(w_{1}\right)$ by colinearity, this collapses to

$$
\left(\epsilon_{61} w_{1}\right)^{\prime}\left(\epsilon_{62} w_{2}\right)^{\prime} \cdots\left(\epsilon_{6,2 n+1} w_{2 n+1}\right)^{\prime}
$$

Since (48) and (49) are each equal to $\pm u_{I^{\prime} J}$, by the uniqueness in Lemma 6.7, $\left(\epsilon_{6 i} w_{i}\right)^{\prime}=\left(\epsilon_{4 i} w_{i}\right)$, which proves 46$)$. 


\section{Cartan factors of Rank 1}

Proof of Proposition 2.6 in the finite dimensional rank 1 case. It follows from Propositions 6.3 and 6.10 that the map $\epsilon(I J) u_{I J} \rightarrow E_{J I}$ is a ternary isomorphism onto $\left(\begin{array}{c}n \\ i_{R}\end{array}\right)$ by $\left(\begin{array}{c}n \\ n-i_{R}+1\end{array}\right)$ complex matrices. By Remark 6.2 and (34), $Y$ is completely isometric to a subtriple $H_{n}^{i_{R}}$ of a Cartan factor of type 1 . In view of Lemma 5.9 this completes the proof of Proposition 2.6 in the case that $Y$ is of type 1 and rank 1 and finite dimensional.

Note that the numbers $n$ and $k$ determine a simple algorithm for constructing the unique matricial space $H_{n}^{k}$ (see the paragraph preceding Examples 1 and 2 below). The spaces $H_{n}^{k}$ are examples of the rank $1 \mathrm{JW}^{*}$-triples whose existence was assumed in section 6 , as the following lemma shows.

Lemma 7.1. The spaces $H_{n}^{k}$ are rank 1 Hilbertian $J C^{*}$-triples with $i_{R}=k$ and $i_{R}+i_{L}=n+1$.

Proof. We will denote the generator $\sum_{I, J} \epsilon(I J) E_{J, c, I}$ of the space $H_{n}^{k}$ by $u_{c}$. Note that the sum is orthogonal by Proposition 6.3 , so the $u_{c}$ are partial isometries. It is essential to notice that, for each $E_{J, c, I}$, there are exactly $k$ (resp. $n-k+1$ ) elements $E_{J^{\prime}, c^{\prime}, I^{\prime}}$ such that $E_{J^{\prime}, c^{\prime}, I^{\prime}}\left[E_{J^{\prime}, c^{\prime}, I^{\prime}}\right]^{*} E_{J, c, I}=E_{J, c, I}$ (resp. $\left.E_{J, c, I}\left[E_{J^{\prime}, c^{\prime}, I^{\prime}}\right]^{*} E_{J^{\prime}, c^{\prime}, I^{\prime}}=E_{J, c, I}\right)$, namely, those $E_{J^{\prime}, c^{\prime}, I^{\prime}}$ with $\left(J^{\prime}-\{c\}\right) \cup\left\{c^{\prime}\right\}=J$ (resp. $\left(I^{\prime}-\{c\}\right) \cup\left\{c^{\prime}\right\}=I$ ). In all other cases $\left[E_{J^{\prime}, c^{\prime}, I^{\prime}}\right]^{*} E_{J, c, I}=0$ (resp. $\left.E_{J, c, I}\left[E_{J^{\prime}, c^{\prime}, I^{\prime}}\right]^{*}=0\right)$. With this in mind, using Proposition 6.3, it is a straightforward verification to show that $\left\{\begin{array}{lll}u_{a} & u_{a} & u_{b}\end{array}\right\}=(1 / 2) u_{b}$, and $\left\{\begin{array}{lll}u_{a} & u_{b} & u_{a}\end{array}\right\}=0$. Lemma 6.9 (a) and the comments preceeding it together with Proposition 6.3 shows easily that $\left\{\begin{array}{lll}u_{a} & u_{b} & u_{c}\end{array}\right\}=0$. Hence, the $H_{n}^{k}$ are rank $1 \mathrm{JC}^{*}$-triples and are thus Hilbertian as discussed at the start of section 5.3.

To see that $k=i_{R}$, consider the expression

$$
u_{r} u_{r}^{*} \cdots u_{2} u_{2}^{*} u_{1}
$$

If $r>k$, then, by the remarks above, for each term $\epsilon(I J) E_{J, 1, I}$ in the expansion of $u_{1}$, there must exist a number $i, 2 \leq i \leq r$ such that $u_{i} u_{i}^{*} \epsilon(I J) E_{J, 1, I}=0$. Hence, (50) is zero. Now assume $r=k$. Suppose $I=\{2, \cdots, r\}$ and $J=\{r+1, \cdots, n\}$. Again by the above remarks, for each $2 \leq i \leq r$, there exists an element $E_{J^{\prime}, i, I^{\prime}}$ in the expansion of $u_{i}$ such that $E_{J^{\prime}, i, I^{\prime}}\left[E_{J^{\prime}, i, I^{\prime}}\right]^{*} E_{J, 1, I}=E_{J, 1, I}$, ensuring at least one nonzero term in the expansion of (50). Since all possible nonzero terms of (50) are $\epsilon(I J) E_{J, 1, I}$ and those are independent, (50) is not zero. It follows that $i_{R}=k$. A similar argument shows that $i_{L}=n-k+1$.

The following lemma implies the statement in Theorem 1 that the $H_{n}^{k}$ are 1mixed injectives.

Lemma 7.2. For each matrix $x=\sum a_{i} u_{i}$ in $H_{n}^{k}$,

$$
\operatorname{tr}\left(\left(x x^{*}\right)^{1 / 2}\right)=\left(\begin{array}{l}
n-1 \\
k-1
\end{array}\right)^{1 / 2}\left(\sum\left|a_{i}\right|^{2}\right)^{1 / 2} .
$$

Proof. We first show that $x x^{*}$ can have at most one nonzero eigenvalue. Indeed, if $x x^{*}$ has at least two distinct nonzero eigenvalues, then we may write $x x^{*}=$ $f\left(x x^{*}\right)+g\left(x x^{*}\right)$ for two nonzero disjointly supported even continuous functions $f$ and $g$ which vanish at zero. Hence, $x x^{*} x=f\left(x x^{*}\right) x+g\left(x x^{*}\right) x$ is a non-trivial orthogonal decomposition of the element $x x^{*} x$ in the rank one $\mathrm{JC}^{*}$-triple $H_{n}^{k}$, which 
is impossible by definition of rank. Hence the eigenvalues of $x x^{*}$ are $\|x\|^{2}=\sum\left|a_{i}\right|^{2}$ and possibly zero.

However, since each $u_{i}$ is the sum of exactly $\left(\begin{array}{l}n-1 \\ k-1\end{array}\right)$ orthogonal matrix units mutiplied by \pm 1 , we have that $\operatorname{tr}\left(x x^{*}\right)=\left(\begin{array}{c}n-1 \\ k-1\end{array}\right) \sum\left|a_{i}\right|^{2}$. Thus the multiplicity of the eigenvalue $\sum\left|a_{i}\right|^{2}$ is $\left(\begin{array}{c}n-1 \\ k-1\end{array}\right)$ and $\operatorname{tr}\left(\left(x x^{*}\right)^{1 / 2}\right)=\left(\begin{array}{c}n-1 \\ k-1\end{array}\right)^{1 / 2}\left(\sum\left|a_{i}\right|^{2}\right)^{1 / 2}$.

Corollary 7.3. The linear map $P$ defined by $P x=\sum \operatorname{tr}\left(x u_{i}^{*} /\left(\begin{array}{c}n-1 \\ k-1\end{array}\right)^{1 / 2}\right) u_{i}$ is a contractive projection from $\left(\begin{array}{l}n \\ k\end{array}\right)$ by $\left(\begin{array}{c}n \\ n-k+1\end{array}\right)$ complex matrices onto $H_{n}^{k}$.

Proof. Let $m$ denote the multiplicity $\left(\begin{array}{c}n-1 \\ k-1\end{array}\right)$. Using Lemma 7.2 and the fact that the $H_{n}^{k}$ are Hilbertian, we see that

$$
\begin{aligned}
\|P x\|^{2} & =\sum\left|\operatorname{tr}\left(x u_{i}^{*} / m^{1 / 2}\right)\right|^{2}=\operatorname{tr}\left(x(P x)^{*}\right) / m^{1 / 2} \\
& \leq\|x\| \operatorname{tr}\left[(P x)(P x)^{*}\right]^{1 / 2} / m^{1 / 2} \\
& =\|x\|\left(\sum\left|\operatorname{tr}\left(x u_{i}^{*} / m^{1 / 2}\right)\right|^{2}\right)^{1 / 2}=\|x\|\|P x\|
\end{aligned}
$$

We now prove Proposition 2.6 in the case that $Y$ is of type 1 and rank 1 and arbitrary dimension. The key to the proof is the following lemma.

Lemma 7.4. Suppose $Y$ is a $J W^{*}$-triple of type 1 and rank 1 with grid $\left\{u_{\lambda}: \lambda \in\right.$ $\Lambda$ \}. Then either $\left(u u^{*}\right)_{I} \neq 0$ for all finite subsets $I \subset \Lambda$, or $\left(u^{*} u\right)_{J} \neq 0$ for all finite subsets $J \subset \Lambda$.

Proof. If $\left(u^{*} u\right)_{I}=0$ for some finite subset $I=\left\{i_{1}, \ldots, i_{n+1}\right\}$ we may assume that $\left(u^{*} u\right)_{\left\{i_{1}, \ldots, i_{n}\right\}} \neq 0$. Then as in (25),

$$
\left(u^{*} u\right)_{\left\{i_{1}, \ldots, i_{n}\right\}}=\left(u^{*} u\right)_{\left\{i_{1}, \ldots, i_{n}-1\right\}} u_{i_{n}}^{*}\left(u u^{*}\right)_{\left\{i_{n}, i_{n}+1, \ldots, m\right\}} u_{i_{n}}
$$

for all $m \geq i_{n}$. Hence $\left(u u^{*}\right)_{\left\{i_{n}, i_{n}+1, \ldots, m\right\}} \neq 0$ for all $m \geq i_{n}$. Then by Lemma 5.8 , $\left(u u^{*}\right)_{J} \neq 0$ for all finite subsets $J \subset \Lambda$.

Proof of Proposition 2.6 in the rank 1 type 1 case. We may assume $\operatorname{dim}(Y)=\infty$. For definiteness, we assume that $\left(u u^{*}\right)_{I} \neq 0$ for all finite subsets $I \subset \Lambda$. The other case in Lemma 7.4 is proved similarly. Let $E_{\lambda}$ denote $1 \otimes \psi_{\lambda}$ in $B(H, \mathbb{C})$, where $\operatorname{dim} H=|\Lambda|$ and $\left\{\psi_{\lambda}\right\}$ is an o.n. basis for $H$. By Proposition 5.10, for all finite subsets $I \subset \Lambda$, the map $\phi\left(u_{\lambda}\right)=E_{\lambda}$ is a complete semi-isometry from $Y_{I}:=\operatorname{sp}\left\{u_{\lambda}: \lambda \in I\right\}$ to $\operatorname{sp}\left\{E_{\lambda}: \lambda \in I\right\}$. As a reflexive space, $Y$ is the norm-closure of the union of all the $Y_{I}$ as $I$ varies over all finite subsets of $\Lambda$, so it follows that $Y$ is completely semi-isometric to $B(H, \mathbb{C})$.

The proofs of Theorems 1, 2 2 and 3(a) being complete, we now finish the proof of Theorem 3, give some examples of the spaces $H_{n}^{k}$, and pose some questions.

Proof of Theorem 3 for the rank 1 case. Let $Y$ be an $n$-dimensional $J W^{*}$-triple of rank 1. It follows from Lemma 5.9 and Proposition 6.10 that $Y=$ $\operatorname{Diag}(p Y,(1-p) Y)$ where $p Y$ and $(1-p) Y$ are triple isomorphic to $Y$, and $p Y$ is completely isometric to some $H_{n}^{i_{R}}$. One now observes that the number $i_{R}$ for 
$(1-p) Y$ is strictly less than the $i_{R}$ for $Y$. Indeed, with $w_{i}=(1-p) u_{i}$, we have

$$
\begin{aligned}
\left(w w^{*}\right)_{\left\{1,2, \ldots, i_{R}\right\}} & =(1-p) u_{i} u_{i}^{*}(1-p) u_{2} u_{2}^{*}(1-p) \cdots(1-p) u_{i_{R}} u_{i_{R}}^{*}(1-p) \\
& =(1-p)\left(u u^{*}\right)_{\left\{1,2, \ldots, i_{R}\right\}} \\
& =\left(1-\sum_{|J|=i_{R}}\left(u u^{*}\right)_{J}\right)\left(u u^{*}\right)_{\left\{1,2, \ldots, i_{R}\right\}} \\
& =\left(u u^{*}\right)_{\left\{1,2, \ldots, i_{R}\right\}}-\left(u u^{*}\right)_{\left\{1,2, \ldots, i_{R}\right\}}=0 .
\end{aligned}
$$

Now set $Y_{1}=Y, p_{1}=p$, and $k_{1}=i_{R}$. Then setting $Y_{2}:=\left(1-p_{1}\right) Y_{1}$ and letting $k_{2}$ denote its $i_{R}$, then $k_{2}<k_{1}$. Continuing in this way we see that $Y=$ $\operatorname{Diag}\left(p_{1} Y_{1}, p_{2} Y_{2}, \ldots, p_{m} Y_{m}\right)$, where each $p_{j} Y_{j}$ is completely isometric to the space $H_{n}^{k_{j}}$. An application of Lemma 2.4 completes the proof of Theorem $3(\mathrm{~b})$.

Note that the spaces Diag $\left(H_{n}^{k_{1}}, \ldots, H_{n}^{k_{m}}\right)$ are examples of Hilbertian rank 1 triples with $i_{R}+i_{L}>n+1$, since $i_{R}=k_{1}, i_{L}=n-k_{m}+1$ and $k_{1}>\ldots>k_{m}$. Note also that the spaces $H_{n}^{i_{R}}$ can be explicitly constructed. Simply index columns (resp. rows) by combinations $I$ (resp. $J$ ) of $\{1, \cdots, n\}$ of length $i_{R}-1$ (resp. $i_{L}-1$ ). Then define an orthonormal basis $\left\{U_{i}\right\}$ for $H_{n}^{i_{R}}$ by the requirement that $U_{i}$ equals the sum of all elements $\epsilon_{I, J} E_{J, I}$ where $I \cap J=\emptyset$ and $(I \cup J)^{c}=i$. Then choose signs $\epsilon_{I, J}$ by the procedure detailed above. We now give some examples.

Example 1. Suppose that $Y=\operatorname{sp}_{\mathbb{C}}\left\{u_{1}, u_{2}, u_{3}\right\}$ and $i_{R}=i_{L}=2$. The rectangular grid given by Proposition 6.10 is depicted by the following array:

\begin{tabular}{cc|ccc} 
& & \multicolumn{4}{|c}{} \\
& & $\{1\}$ & \{ & $\{2\}$ \\
\hline \multirow{3}{*}{$J$} & $\{1\}$ & $u_{2} u_{1}^{*} u_{3}$ & $u_{2} u_{2}^{*} u_{3} u_{1}^{*} u_{1}$ & $-u_{3} u_{3}^{*} u_{2} u_{1}^{*} u_{1}$ \\
& $\{2\}$ & $-u_{1} u_{1}^{*} u_{3} u_{2}^{*} u_{2}$ & $-u_{1} u_{2}^{*} u_{3}$ & $u_{3} u_{3}^{*} u_{1} u_{2}^{*} u_{2}$ \\
& $\{3\}$ & $u_{1} u_{1}^{*} u_{2} u_{3}^{*} u_{3}$ & $-u_{2} u_{2}^{*} u_{1} u_{3}^{*} u_{3}$ & $u_{1} u_{3}^{*} u_{2}$
\end{tabular}

By (34) the ternary isomorphism from the span of this rectangular grid to the canonical grid in $B\left(\mathbb{C}^{3}\right)$, when restricted to $Y$, satisfies

$$
u_{1} \mapsto\left[\begin{array}{rrr}
0 & 0 & 0 \\
0 & 0 & 1 \\
0 & -1 & 0
\end{array}\right] \quad, \quad u_{2} \mapsto\left[\begin{array}{rrr}
0 & 0 & -1 \\
0 & 0 & 0 \\
1 & 0 & 0
\end{array}\right] \quad, \quad u_{3} \mapsto\left[\begin{array}{rrr}
0 & 1 & 0 \\
-1 & 0 & 0 \\
0 & 0 & 0
\end{array}\right]
$$

Thus $H_{3}^{2}$ is the subtriple of $B\left(\mathbb{C}^{3}\right)$ consisting of all matrices of the form

$$
\left[\begin{array}{rrr}
0 & a & -b \\
-a & 0 & c \\
b & -c & 0
\end{array}\right]
$$

and hence in this case $Y$ is actually completely semi-isometric to the Cartan factor $A\left(\mathbb{C}^{3}\right)$ of 3 by 3 anti-symmetric complex matrices. 
Example 2. Suppose that $Y=\operatorname{sp}_{\mathbb{C}}\left\{u_{1}, u_{2}, u_{3}, u_{4}\right\}$ and $i_{R}=3, i_{L}=2$. The rectangular grid given by Proposition 6.10 is depicted by the following array:

\begin{tabular}{rr|rrrrrr} 
& & \multicolumn{5}{|c}{$I$} \\
\hline \multirow{2}{*}{$J$} & $\{1\}$ & 22314 & $\{1,3\}$ & $\{1,4\}$ & $\{2,3\}$ & $\{2,4\}$ & $\{3,4\}$ \\
\hline & $\{2\}$ & 11324 & 1133422 & -1144322 & 33124 & -44123 & 3344122 \\
& $\{3\}$ & -1122433 & -11234 & 1144233 & 22134 & -2244133 & 44132 \\
& $\{4\}$ & 1122344 & -1133244 & 11243 & 2233144 & -22143 & 33142
\end{tabular}

Here we have used the abbreviation 22314 for $u_{2} u_{2}^{*} u_{3} u_{1}^{*} u_{4}$ and so forth.

By (34) the ternary isomorphism from the span of this rectangular grid to the canonical grid in $B\left(\mathbb{C}^{6}, \mathbb{C}^{4}\right)$, when restricted to $Y$, satisfies

$$
u_{1} \mapsto\left[\begin{array}{rrrrrr}
0 & 0 & 0 & 0 & 0 & 0 \\
0 & 0 & 0 & 0 & 0 & 1 \\
0 & 0 & 0 & 0 & -1 & 0 \\
0 & 0 & 0 & 1 & 0 & 0
\end{array}\right] \quad, \quad u_{2} \mapsto\left[\begin{array}{rrrrrr}
0 & 0 & 0 & 0 & 0 & -1 \\
0 & 0 & 0 & 0 & 0 & 0 \\
0 & 0 & 1 & 0 & 0 & 0 \\
0 & -1 & 0 & 0 & 0 & 0
\end{array}\right]
$$

and

$$
u_{3} \mapsto\left[\begin{array}{rrrrrr}
0 & 0 & 0 & 0 & 1 & 0 \\
0 & 0 & -1 & 0 & 0 & 0 \\
0 & 0 & 0 & 0 & 0 & 0 \\
1 & 0 & 0 & 0 & 0 & 0
\end{array}\right] \quad, \quad u_{4} \mapsto\left[\begin{array}{rrrrrr}
0 & 0 & 0 & -1 & 0 & 0 \\
0 & 1 & 0 & 0 & 0 & 0 \\
-1 & 0 & 0 & 0 & 0 & 0 \\
0 & 0 & 0 & 0 & 0 & 0
\end{array}\right],
$$

so that $Y$ is completely semi-isometric to $H_{4}^{3}$, which is the subtriple of $B\left(\mathbb{C}^{6}, \mathbb{C}^{4}\right)$ consisting of all matrices of the form

$$
\left[\begin{array}{rrrrrr}
0 & 0 & 0 & -d & c & -b \\
0 & d & -c & 0 & 0 & a \\
-d & 0 & b & 0 & -a & 0 \\
c & -b & 0 & a & 0 & 0
\end{array}\right]
$$

We now show that $H_{3}^{2}$ is not completely semi-isometric to $R_{3}$, as suggested to us by N. Ozawa. It is clear that similar arguments can be used to prove Theorem 1 (d). Since $R_{3}$ is a homogeneous operator space, if there were a complete semi-isometry of $H_{3}^{2}$ onto $R_{3}$, then every isometry from $H_{3}^{2}$ onto $R_{3}$ would be a complete semiisometry. In the notation of Example 1, let $U: H_{3}^{2} \rightarrow R_{3} \subset M_{3}(\mathbb{C})$ be the isometry defined by

$$
\begin{aligned}
& {\left[\begin{array}{rrr}
0 & 0 & 0 \\
0 & 0 & 1 \\
0 & -1 & 0
\end{array}\right] \mapsto\left[\begin{array}{rrr}
0 & 0 & 1 \\
0 & 0 & 0 \\
0 & 0 & 0
\end{array}\right],\left[\begin{array}{rrr}
0 & 0 & -1 \\
0 & 0 & 0 \\
1 & 0 & 0
\end{array}\right] \mapsto\left[\begin{array}{lll}
0 & 1 & 0 \\
0 & 0 & 0 \\
0 & 0 & 0
\end{array}\right], } \\
& {\left[\begin{array}{rrr}
0 & 1 & 0 \\
-1 & 0 & 0 \\
0 & 0 & 0
\end{array}\right] \mapsto\left[\begin{array}{lll}
1 & 0 & 0 \\
0 & 0 & 0 \\
0 & 0 & 0
\end{array}\right] . }
\end{aligned}
$$

Then $U$ is not a complete contraction, since

$$
\left\|\left[\begin{array}{rrrrrrrrr}
0 & -1 & 0 & 0 & 0 & -1 & 0 & 0 & 0 \\
1 & 0 & 0 & 0 & 0 & 0 & 0 & 0 & 1 \\
0 & 0 & 0 & 1 & 0 & 0 & 0 & -1 & 0
\end{array}\right]\right\|=\sqrt{2}
$$


and

$$
\left\|\left[\begin{array}{lllllllll}
1 & 0 & 0 & 0 & 1 & 0 & 0 & 0 & 1 \\
0 & 0 & 0 & 0 & 0 & 0 & 0 & 0 & 0 \\
0 & 0 & 0 & 0 & 0 & 0 & 0 & 0 & 0
\end{array}\right]\right\|=\sqrt{3}
$$

Problem 1. What is the completely bounded Banach-Mazur distance $d_{c b}\left(H_{n}^{k}, R_{n}\right)$ ?

Problem 2. What can one say about an arbitrary 1-mixed injective operator space? What can one say about an arbitrary $J W^{*}$-triple up to complete isometry?

Remark 7.5. The authors hope to classify all 1-mixed injectives possessing a predual in a future publication by using the known structure theory of $J B W^{*}$-triples in [18] and 20].

Remark 7.6. After completing this paper, the authors discovered that the spaces $H_{n}^{k}$ appear, in a slightly different form, in [1] in their solution to the contractive projection problem on the compact operators on a separable Hilbert space. Their methods and proofs are different from ours. In the special case that the projection is weak ${ }^{*}$-weak ${ }^{*}$ continuous and $H$ is separable, Theorem 2 can be derived from their results.

\section{REFERENCES}

[1] J. Arazy and Y. Friedman, Contractive projections in $C_{1}$ and $C_{\infty}$, Mem. Amer. Math. Soc. 13 (1978), no 200.

[2] T. J. Barton, T. C. Dang and G. Horn, Normal representations of Banach Jordan triple systems, Proc. Amer. Math. Soc. 102 (1988), 551-555.

[3] T. J. Barton and R. Timoney, Weak*-continuity of Jordan triple products and its applications, Math. Scand. 59 (1986), 177-191.

[4] D. Blecher and V. Paulsen, Tensor products of operator spaces, J. Funct. Anal. 99 (1992), 262-292.

[5] O. Bratteli and D. W. Robinson, Operator algebras and quantum statistical mechanics II, Springer-Verlag, 1981.

[6] M.D. Choi and E. Effros, Injectivity and operator spaces, J. Funct. Anal. 24 (1977), 156-209.

[7] E. Christensen and A. M. Sinclair, Completely bounded isomorphisms of injective von Neumann algebras, Proc. Edin. Math. Soc. 32 (1989), 317-327.

[8] T. Dang and Y. Friedman, Classification of JBW*-triple factors and applications, Math. Scand. 61 (1987), 292-330.

[9] E. Effros and Z. J. Ruan, On matrically normed spaces, Pac. J. Math. 132 (1988), 243-264.

[10] E. Effros and Z. J. Ruan, Operator Spaces, Oxford University Press, 2000.

[11] E. Effros and E. Stormer, Positive projections and Jordan structure in operator algebras, Math. Scand. 45 (1979), 127-138.

[12] Y. Friedman and B. Russo, Contractive projections on operator triple systems, Math. Scand. 52 (1983), 279-311.

[13] Y. Friedman and B. Russo, Solution of the contractive projection problem, J. Funct. Anal. 60 (1985), 56-79.

[14] Y. Friedman and B. Russo, Structure of the predual of a $J B W^{*}$-triple, J. Reine Angew. Math. 356 (1985), 67-89.

[15] H. Hanche-Olsen and E. Stormer, Jordan Operator Algebras, Pitman, 1984.

[16] L. A. Harris, Bounded symmetric domains in infinite dimensional spaces. In: Hayden,T. L. ,Suffridge,T. J. (eds.) Infinite dimensional holomorphy. Proceedings, 1973 (Lecture Notes in Mathematics 364,pp. 13-40), Springer 1974.

[17] M. Hestenes, A ternary algebra with applications to matrices and linear transformations, Arch. Rational Mech. Anal. 11 (1962), 138-194.

[18] G. Horn, Classification of JBW*-triples of Type I, Math. Zeit. 196 (1987), 271-291. 
[19] G. Horn, Coordinatization theorems for JBW*-triples, Quart. J. Math. 38 (1987), 321-335.

[20] G. Horn and E. Neher, Classification of continuous JBW*-triples, Trans. Amer. Math. Soc. 306 (1987), 553-578.

[21] W. Kaup, A Riemann mapping theorem for bounded symmetric domains in complex Banach spaces, Math. Zeit. 183 (1983), 503-529.

[22] W. Kaup, Contractive projections on Jordan $C^{*}$-algebras and generalizations, Math. Scand. 54 (1984), 95-100.

[23] O. Loos, Jordan Pairs Lecture Notes in Mathematics 460, Springer 1975.

[24] O. Loos, Bounded symmetric domains and Jordan pairs, Lecture Notes, Univ. of California, Irvine, 1977.

[25] M. Neal and B. Russo, Contractive projections and operator spaces, C. R. Acad. Sci. Paris 331 (2000), 873-878.

[26] E. Neher, Jordan triple systems by the grid approach, Lecture Notes in Mathematics 1280, Springer, 1987.

[27] T. Oikhberg and H. P. Rosenthal, Extension properties for the space of compact operators, J. Funct. Anal. 179 (2001), 251-308.

[28] G. Pisier, The operator Hilbert space $\mathrm{OH}$, complex interpolation and tensor norms, Mem. Amer. Math. Soc. 122 (1996), no 585.

[29] G. Pisier, Noncommutative vector-valued $L_{p}$-spaces and completely p-summing maps, Astérisque No. 247, Soc. Math. France, 1998

[30] A. G. Robertson, Injective matricial Hilbert spaces, Math. Proc. Camb. Philos. Soc. 110 (1991), no. 1, 183-190.

[31] A. G. Robertson and S. Wasserman, Completely bounded isomorphisms of injective operator systems, Bull. Lon. Math. Soc. 21 (1989), 285-290.

[32] A. G. Robertson and M. A. Youngson, Isomorphisms of injective operator spaces and Jordan triple systems, Quart. J. Math. 41 (1990), 449-462.

[33] Z.-J. Ruan, Subspaces of $C^{*}$-algebras, J. Funct. Anal. 76 (1988), 217-230.

[34] Z. -J. Ruan, Injectivity of operator spaces, Trans. Amer. Math. Soc. 315 (1989), 89-104.

[35] B. Russo, Structure of JB*-triples, In: Jordan Algebras, Proceedings of the Oberwolfach Conference 1992, Eds: W. Kaup,K. McCrimmon,H. Petersson, de Gruyter, Berlin (1994), 209-280.

[36] S. Sakai, $C^{*}$-algebras and $W^{*}$-algebras, Ergebnisse der Mathematik und ihrer Grenzgebiete 60, Springer-Verlag, 1971

[37] H. Upmeier, Symmetric Banach manifolds and Jordan $C^{*}$-algebras, North-Holland Publishing Co. , Amsterdam-New York, 1985.

[38] M. A. Youngson, Completely contractive projections on $C^{*}$-algebras, Quart. J. Math. 34 (1983), 507-511.

[39] H. Zettl, A characterization of ternary rings of operators, Adv. Math. 48 (1983), 117-143.

Department of Mathematics, University of California, Irvine, California 92697-3875

E-mail address: mneal@math.uci.edu brusso@math.uci.edu 